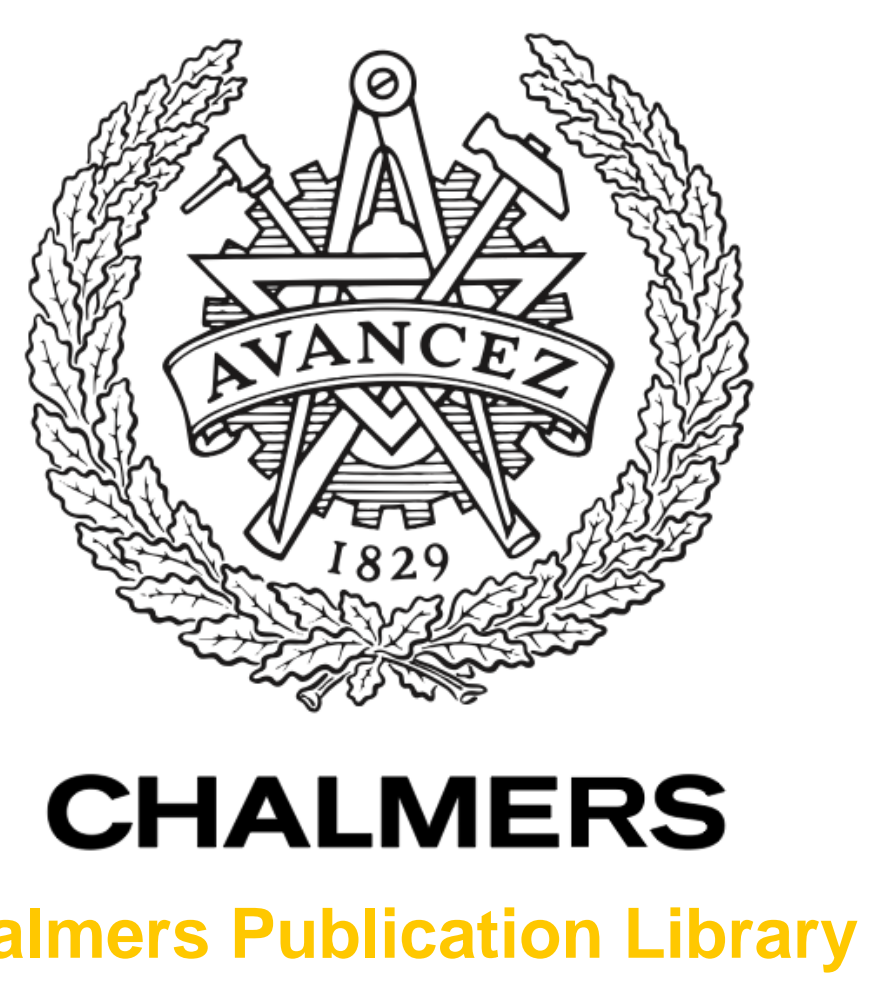

Chalmers Publication Library

\title{
Modelling of 3D fields due to ferritic inserts and test blanket modules in toroidal geometry at ITER
}

This document has been downloaded from Chalmers Publication Library (CPL). It is the author's version of a work that was accepted for publication in:

Nuclear Fusion (ISSN: 0029-5515)

Citation for the published paper:

Liu, Y. ; Akaslompolo, S. ; Cavinato, M. et al. (2016) "Modelling of 3D fields due to ferritic inserts and test blanket modules in toroidal geometry at ITER". Nuclear Fusion, vol. 56(6), pp. Art. no. 066001.

http://dx.doi.org/10.1088/0029-5515/56/6/066001

Downloaded from: http://publications.lib.chalmers.se/publication/238352

Notice: Changes introduced as a result of publishing processes such as copy-editing and formatting may not be reflected in this document. For a definitive version of this work, please refer to the published source. Please note that access to the published version might require a subscription.

Chalmers Publication Library (CPL) offers the possibility of retrieving research publications produced at Chalmers University of Technology. It covers all types of publications: articles, dissertations, licentiate theses, masters theses, conference papers, reports etc. Since 2006 it is the official tool for Chalmers official publication statistics. To ensure that Chalmers research results are disseminated as widely as possible, an Open Access Policy has been adopted.

The CPL service is administrated and maintained by Chalmers Library. 


\title{
Modelling of 3D fields due to ferritic inserts and test blanket modules in toroidal geometry in ITER
}

Yueqiang Liu ${ }^{1,2,3}$, Simppa Äkäslompolo ${ }^{4}$, Mario Cavinato ${ }^{5}$, Florian Koechl ${ }^{1}$, Taina KurkiSuonio $^{4}$, Li Li ${ }^{6,7}$, Vassili Parail ${ }^{1}$, Gabriella Saibene ${ }^{5}$, Konsta Särkimäki ${ }^{4}$, Seppo Sipilä ${ }^{4}$, Jari Varje $^{4}$

${ }^{1}$ CCFE, Culham Science Centre, Abingdon, OX14 3DB, UK

2 Southwestern Institute of Physics, PO Box 432, Chengdu 610041, China

${ }^{3}$ Department of Earth and Space Science, Chalmers University of Technology, SE-412 96 Gothenburg, Sweden

${ }^{4}$ Department of Applied Physics, Aalto University, FI-00076 AALTO, Finland

${ }^{5}$ Fusion for Energy, Torres Diagonal Litoral, Edificio B3, 08019 Barcelona, Spain

${ }^{6}$ Donghua University, Shanghai, China

${ }^{7}$ Forschungszentrum Jülich $\mathrm{GmbH}$, Institut för Energie-und Klimaforschung-Plasmaphysik, Jülich, Germany

E-mail: yueqiang.liu@ccfe.ac.uk

\begin{abstract}
Computations in toroidal geometry are systematically performed for the plasma response to 3D magnetic perturbations, produced by ferritic inserts (FIs) and test blanket modules (TBMs), for four ITER plasma scenarios: the 15MA baseline, the 12.5MA hybrid, the 9MA steady state, and the 7.5MA half-field Helium plasma. Due to broad toroidal spectrum of the FI and TBM fields, the plasma response for all the $n=1-6$ field components are computed and compared. The plasma response is found to be weak for the high- $n(n>4)$ components. The response is globally not sensitive to the toroidal plasma flow speed, as long as the latter is not reduced by an order of magnitude. This is essentially due to the strong screening effect occuring at a finite flow as predicted for ITER plasmas. The ITER error field correction coils (EFCC) are used to compensate the $n=1$ field errors produced by FIs and TBMs for the baseline scenario, for the purpose of avoiding the mode locking. It is found that the middle row of EFCC, with a suitable toroidal phase for the coil current, can provide the best correction of these field errors, according to various optimization criteria. On the other hand, even without correction, it is predicted that these $n=1$ field errors do not cause substantial flow damping for the 15MA baseline scenario.
\end{abstract}

\section{Introduction}

It is well known that periodic toroidal field ripples can have negative effects on the plasma performance, in particular in H-mode plasmas [1,2]. Fast ion loss due to the ripple fields is one of the significant concerns in ITER and future fusion reactors. It is by this reason, ferritic inserts (FIs) have been designed for ITER, in order to reduce the ripple fields in ITER, which has predominantly the $n=18$ ( $n$ is the toroidal mode number) component due to symmetry of the toroidal field coils. While cancelling the ripple fields, these FIs produce small, but not negligible field errors, of other toroidal mode numbers. 
Test blanket modules (TBMs), which are not periodically distributed along the toroidal angle of the torus, are also designed for ITER. These TBMs contain massive ferromagnetic materials (several tons), which again generate 3D error fields (EFs) in ITER. Unlike the present devices, where the EFs can be minimized by careful alignment of the machine and the coils system, the EFs in ITER, as well as their correction, are a substantial concern, partly due to the presence of TBMs. In particular, a broadband toroidal spectrum of the EFs is expected in ITER, due to the non-periodic distribution of TBMs.

Recent TBM experiments, carried out in DIII-D [3,4] using mock up coils, have shown that, whilst with little effect on the L-mode plasmas and with moderate impact (up to 20\%) on the particle and energy confinement in H-mode plasmas, the TBM coils do have significant effect on the plasma stability, in particular on the plasma flow damping and the subsequent mode locking in DIII-D. It is therefore of critical importance to investigate the TBM induced mode locking in ITER. It should also be mentioned that the vacuum magnetic field, produced by the mock up coils in DIII-D, is about 3 times larger than that predicted by the ITER TBM.

Understanding the structure of the vacuum fields, produced by FIs and TBMs, is certainly an important first step. However, as has been recently realized, taking into account the plasma response to the 3D external fields may be crucial. This is useful not only just for understanding the modification (plasma shielding and/or amplification) of the field structure due to the plasma response, but also, and perhaps even more importantly, for providing better guidance of correcting these field errors, for understanding the potential mode locking induced by the low- $n$ components of these fields, and for further investigation of energetic particle losses in the presence of the total 3D field perturbations including the plasma response [5].

Various physics models have recently been developed and applied to model the plasma response to external 3D fields. In particular, ideal single fluid plasma response model has been used to guide the error field correction in ITER (not including EFs resulting from the FIs and TBMs, though) [6], ideal/resistive single fluid models have been successfully applied to simulate the resonant magnetic perturbation (RMP) experiments in DIII-D [7, 8, 9, 10, 11], MAST [12], and ASDEX Upgrade [13]. A magnetohydrodynamic-kinetic hybrid model has been shown to quantitatively reproduce the response of plasmas with pressure approaching or even exceeding the the no-wall limit for the external ideal kink instability [14]. Two-fluid model has been assumed to compute the plasma response in DIII-D [15]. Successful comparisons have been made between various models and experiments [9].

In this work, we use the MARS-F/K/Q codes suite $[16,17,18]$ to model the plasma response due to ripples, FIs, TBMs, and for limited cases also with the inclusion of the fields from edge localized mode (ELM) control coils. The plasma response to the ELM coils has previously been more systematically modelled for ITER $[12,27]$. We shall consider four ITER scenarios - the 15MA baseline, the 12.5MA hybrid, the 9MA steady state, and finally the 7.5MA halffield Helium plasma. Three issues are addressed: (i) the linear plasma response to various decompositions of the vacuum fields, of different toroidal mode numbers $n=1-6$, for all four scenarios; (ii) optimal correction of the $n=1$ field errors due to FIs and TBMs, using the ITER error field correction coils (EFCC), for the 15MA baseline scenario, for the purpose of avoiding mode locking; (iii) simulation of the plasma flow damping due to the $n=1$ FIs and TBMs fields for the 15MA plasma, using both the quasi-linear MARS-Q code, and the 
Section 2 briefly describes the MARS-F/K/Q models, followed by discussions on the input data for the modelling - primarily the equilibrium specifications for four ITER scenarios in Section 3. Section 4 reports the systematic study of the linear plasma response, for the four ITER scenarios from Section 3. Section 5 reports the modelling results on the $n=1$ error field correction (EFC). Section 6 reports MARS-Q and JINTRAC results on the plasma toroidal momentum confinement in the presence of the $n=1$ FIs and TBMs fields. Section 7 summarizes the work.

\section{The MARS-F/K/Q model for computing plasma response}

We compute the linear plasma response in the framework of the single fluid, resistive MHD approximation. The plasma model, with a given toroidal rotation $\mathbf{V}_{0}=R \Omega \hat{\phi}$, is thus described by the following set of equations

$$
\begin{aligned}
i\left(\Omega_{\mathrm{EF}}+n \Omega\right) \xi= & \mathbf{v}+(\xi \cdot \nabla \Omega) R \hat{\phi} \\
i \rho\left(\Omega_{\mathrm{EF}}+n \Omega\right) \mathbf{v}= & -\nabla p+\mathbf{j} \times \mathbf{B}+\mathbf{J} \times \mathbf{b}-\rho[2 \Omega \hat{\mathbf{Z}} \times \mathbf{v}+(\mathbf{v} \cdot \nabla \Omega) R \hat{\phi}] \\
& -\rho \kappa_{\|}\left|k_{\|} v_{t h, i}\right|\left[\mathbf{v}+(\xi \cdot \nabla) \mathbf{V}_{0}\right]_{\|}, \\
i\left(\Omega_{\mathrm{EF}}+n \Omega\right) \mathbf{b}= & \nabla \times(\mathbf{v} \times \mathbf{B})+(\mathbf{b} \cdot \nabla \Omega) R \hat{\phi}-\nabla \times(\eta \mathbf{j}), \\
i\left(\Omega_{\mathrm{EF}}+n \Omega\right) p= & -\mathbf{v} \cdot \nabla P-\Gamma P \nabla \cdot \mathbf{v}, \\
\mathbf{j}= & \nabla \times \mathbf{b},
\end{aligned}
$$

where $R$ is the plasma major radius, $\hat{\phi}$ the unit vector along the geometric toroidal angle $\phi$ of the torus, $\hat{\mathbf{Z}}$ the unit vector in the vertical direction in the poloidal plane. $\Omega_{\mathrm{EF}}$ is the excitation frequency of the external (to the plasma) 3D field perturbations. In this work, these 3D fields, which are generally referred to here as the error field (EF), are caused by ripple fields, FI, TBM, as well as edge localized mode (ELM) control coils. We assume that these 3D fields are generally dc fields, with $\Omega_{\mathrm{EF}}=0 . n$ is the toroidal harmonic number. For a linear response of axi-symmetric equilibria, we need to consider a single $n$ only. The plasma resistivity is denoted by $\eta$. The variables $\xi, \mathbf{v}, \mathbf{b}, \mathbf{j}, p$ represent the plasma displacement, perturbed velocity, magnetic field, current, and pressure, respectively. The equilibrium plasma density, field, current, and pressure are denoted by $\rho, \mathbf{B}, \mathbf{J}, P$, respectively.

The last term in Eq. (2) describes the effect of parallel sound wave damping [20], where $\kappa$ is a numerical coefficient determining the damping "strength". $k_{\|}=(n-m / q) / R$ is the parallel wave number, with $m$ being the poloidal harmonic number and $q$ being the safety factor. $v_{t h, i}=$ $\sqrt{2 T_{i} / M_{i}}$ is the thermal ion velocity, with $T_{i}, M_{i}$ being the thermal ion temperature and mass, respectively. The parallel component of the perturbed velocity is taken along the equilibrium field line. In this work, we assume $\kappa_{\|}=1.5$, corresponding to a strong sound wave damping, which has been shown to be adequate for modelling the low beta plasma response to external $3 \mathrm{D}$ fields $[12,13]$.

The external 3D field is normally generated by the source current, or an equivalent surface 
current $\mathbf{j}_{\text {ESC, }}$ located in the vacuum region outside the plasma

$$
\nabla \times \mathbf{b}=\mathbf{j}_{\mathrm{ESC}}, \quad \nabla \cdot \mathbf{j}_{\mathrm{ESC}}=0 .
$$

In this study, the source current is specified as a surface current, following a rigorous procedure as described in Ref. [21].

Note that for plasma response modelling, we also make use of the divergence-free condition for the total field perturbation $\mathbf{b}$ in the plasma region, by replacing one of the equations in the Ohm's law (3) by $\nabla \cdot \mathbf{b}=0$. This is to ensure that the field divergence-free condition is numerically enforced. The plasma-vacuum interface conditions are the continuity of the normal component of the field $\mathbf{b}$, and the (total) perturbed pressure balance condition. The former is satisfied automatically by solving for the total $\mathbf{b}$ field across all regions.

All the perturbed quantities are decomposed into Fourier harmonics along the toroidal and poloidal angles of the torus. For linear perturbations, we solve Eqs. (1)-(5) for each toroidal harmonic $n$ separately. For each given $n$, all the poloidal harmonics, however, couple together, and need to be included into the solution at the same time. Along the radial direction, Eqs. (1)-(5) are solved using the finite element method.

Details of the drift kinetic extension of the above model are described in Ref. [17, 22]. This is essentially a MHD-kinetic hybrid model, based on the so called non-perturbative approach. The code implementation (MARS-K) benchmarking results were reported in [23] and validated against experiments [24, 14]. The quasi-linear extension, implemented to model the external 3D field induced toroidal flow damping of the plasma, was reported in [18]. Various momentum sink terms associated with 3D field perturbations, including the electromagnetic resonant $(\mathbf{j} \times \mathbf{b})$ torque, the neoclassical toroidal viscous (NTV) torque, as well as the torque due to the Reynolds stress (REY), have been implemented into MARS-Q, benchmarked [25] and validated [26].

The MARS-F/K/Q models have been extensively used to study the plasma response in DIII-D $[7,8,9,28,10,14,11]$, NSTX [24], MAST [12, 26], ASDEX Upgrade [13], as well as ITER [27].

\section{Specification of ITER equilibria and 3D vacuum fields}

The main input data for the MARS-F/K/Q modelling are (i) the plasma equilibria, and (ii) the external perturbed vacuum 3D magnetic fields. In this study, four plasma scenarios are defined for ITER, as listed in Tab. 1. For the baseline and the hybrid scenario, two phases are considered - the plasma current ramp-up phase and the current flat-top phase.

These four ITER plasma scenarios, which will be considered for the plasma response computations, are the $15 \mathrm{MA}$ inductive scenario at $\mathrm{Q}=10$ and at the full field (5.3T), at one time slice during current ramp-up (RU, with $I_{p}=12 \mathrm{MA}$ ) and one time slice during current flat-top (FT); the $12.5 \mathrm{MA}$ hybrid scenario at full field, at one time slice during current ramp-up $\left(I_{p}=10 \mathrm{MA}\right)$ 
Table 1: Plasma scenarios considered in the study.

\begin{tabular}{|l|l|l|l|l|}
\hline Scenario & $B_{0}[\mathrm{~T}]$ & $I_{p}[\mathrm{MA}]$ & Ref.\# & FT or RU \\
\hline \hline Baseline & 5.3 & 15 & 10470 & Flat-Top \\
\cline { 4 - 5 } & & & 10060 & Ramp-Up \\
\hline Hybrid & 5.3 & 12.5 & 13090 & Flat-Top \\
\cline { 4 - 5 } & & & 13050 & Ramp-Up \\
\hline Steady-State & 5.3 & 9 & 10100 & Flat-Top \\
\hline Half-Field Helium & 2.65 & 7.5 & 10920 & Flat-Top \\
\hline
\end{tabular}

and one time slice during current flat-top; the 9MA steady state scenario at full field, at one time slice during the steady state phase. the half-field (2.65T) and half-current (7.5MA) scenario with the helium plasma, at one time slice during current flat-top. These equilibria were produced by the JINTRAC transport code [19].

Figures 1 and 2 show two examples of the equilibrium profiles, for the 15MA baseline scenario and the 9MA steady state scenario, respectively. Both equilibria shown here are in the current FT phase. For the baseline plasma, the safety factor $q_{95}$ is 3.23. The normalized plasma pressure is $\beta_{N}=2.04$. Transport modelling produces two toroidal rotation profiles, depending on the assumption on the Prandtl number $\operatorname{Pr}$ (the ratio of the momentum diffusivity to the thermal diffusivity). For the 9MA plasma, the $q_{95}$ value is 5.84. The normalized plasma pressure is $\beta_{N}=2.87$.

In order to compute the plasma response, the external 3D fields, caused by the toroidal ripples, FIs or TBMs, have to be properly specified and included into the MARS-F model. These vacuum fields, including also additional, not small contributions from irregular neutral beam ports in ITER, are computed by a combined finite element - Biot-Savart law integrator method [29]. Based on these vacuum fields, a rigorous procedure, which is valid in a generic toroidal geometry, has been devised [21] to compute the plasma response fields. This procedure relies on computing the equivalent surface current (ESC), which is a current that produces exactly the same vacuum field inside a virtual surface (VS), shown by the solid line in Fig. 3, as that of the external 3D field. Thus the eventual plasma response computation is converted to the response of the plasma to the ESC.

The above ESC procedure is strictly valid only if the external field sources (e.g. a current source) are not perturbed by the plasma response. In our study, the external field sources (FI and TBM) are ferromagnetic, and therefore will eventually react to the pure plasma response field produced by the perturbed plasma current. This effect is neglected in this work, since, as will be shown later, the pure plasma response field is generally small compared to the applied vacuum external field for these ITER plasmas. It is possible to design a more involved procedure that takes into account this secondary effect, similar to the so called backward coupling scheme as envisaged in Refs. [30, 31], where stability problems (instead of the response problem) were considered, and where the influence of the plasma response on the external structures (the conducting walls) is rigorously taken into account. 

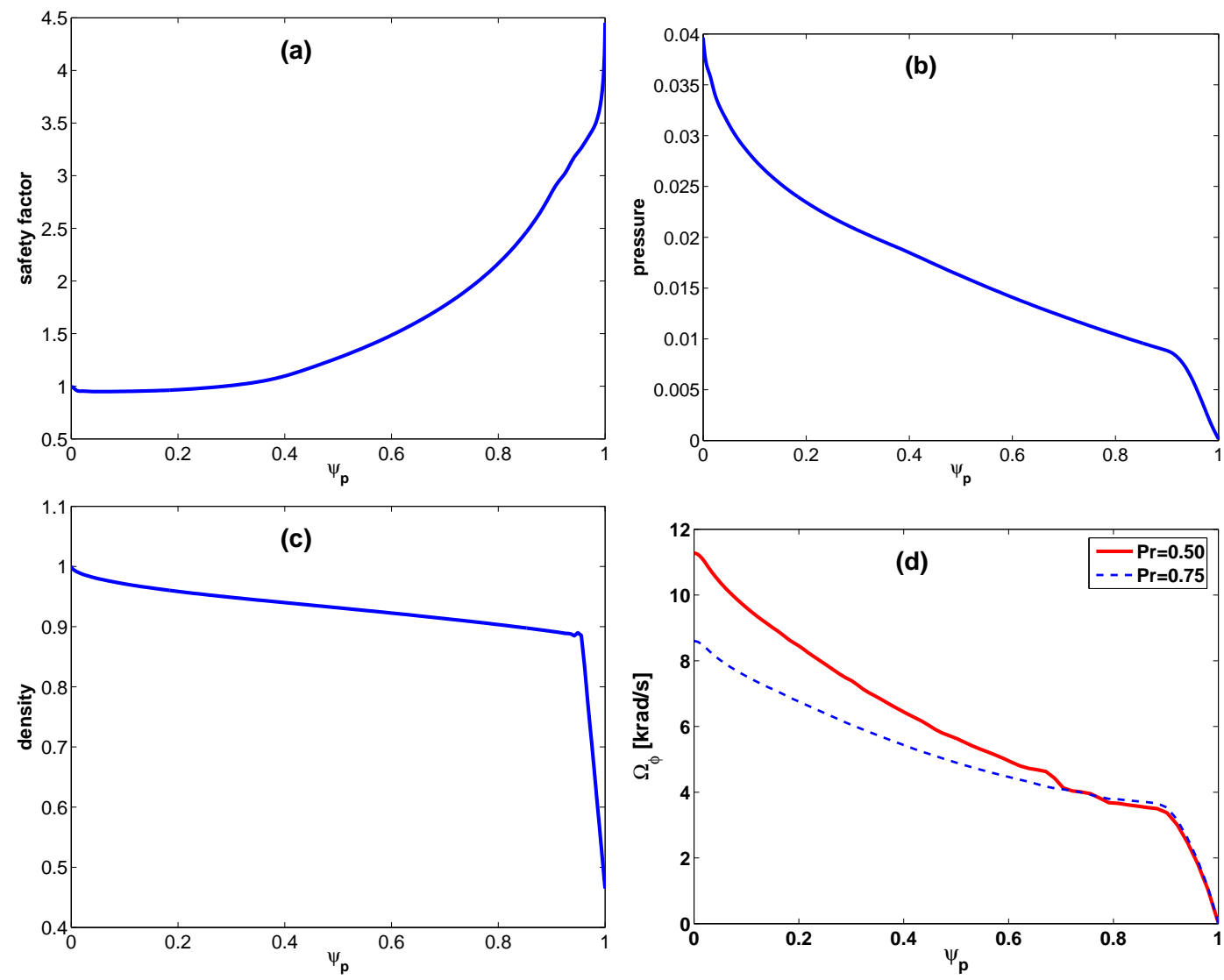

Figure 1: Equilibrium radial profiles of (a) the safety factor $q$, (b) pressure normalized by $B_{0}^{2} / \mu_{0}$, (c) plasma density normalized to unity at the magnetic axis, and (d) toroidal rotation frequency, for the modelled 15MA baseline plasma at the current flat-top phase. 

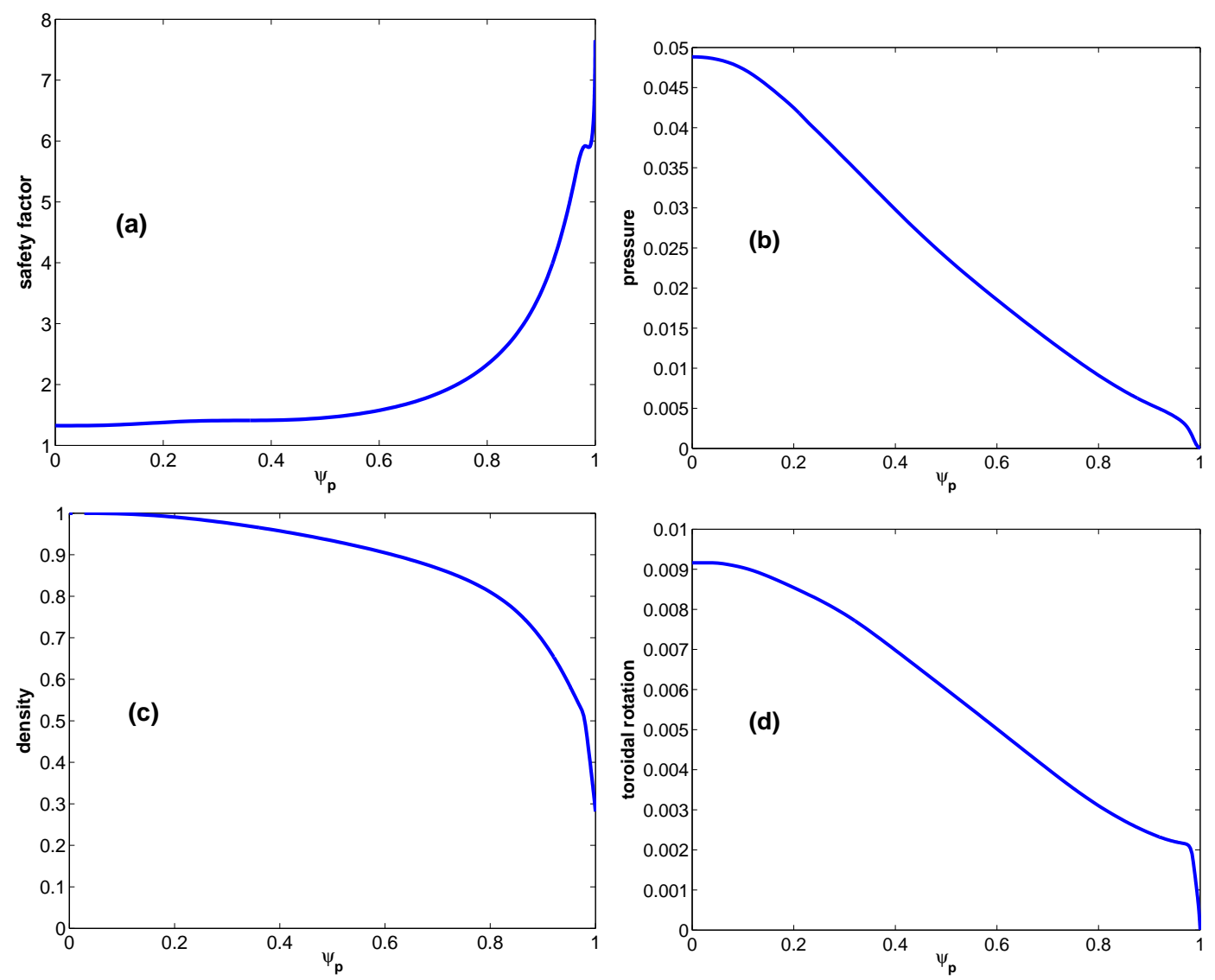

Figure 2: Equilibrium radial profiles of (a) the safety factor $q$, (b) pressure normalized by $B_{0}^{2} / \mu_{0}$, (c) plasma density normalized to unity at the magnetic axis, and (d) toroidal rotation frequency normalized by the on-axis toroidal Alfvén frequency, for the modelled 9MA steady state plasma. 


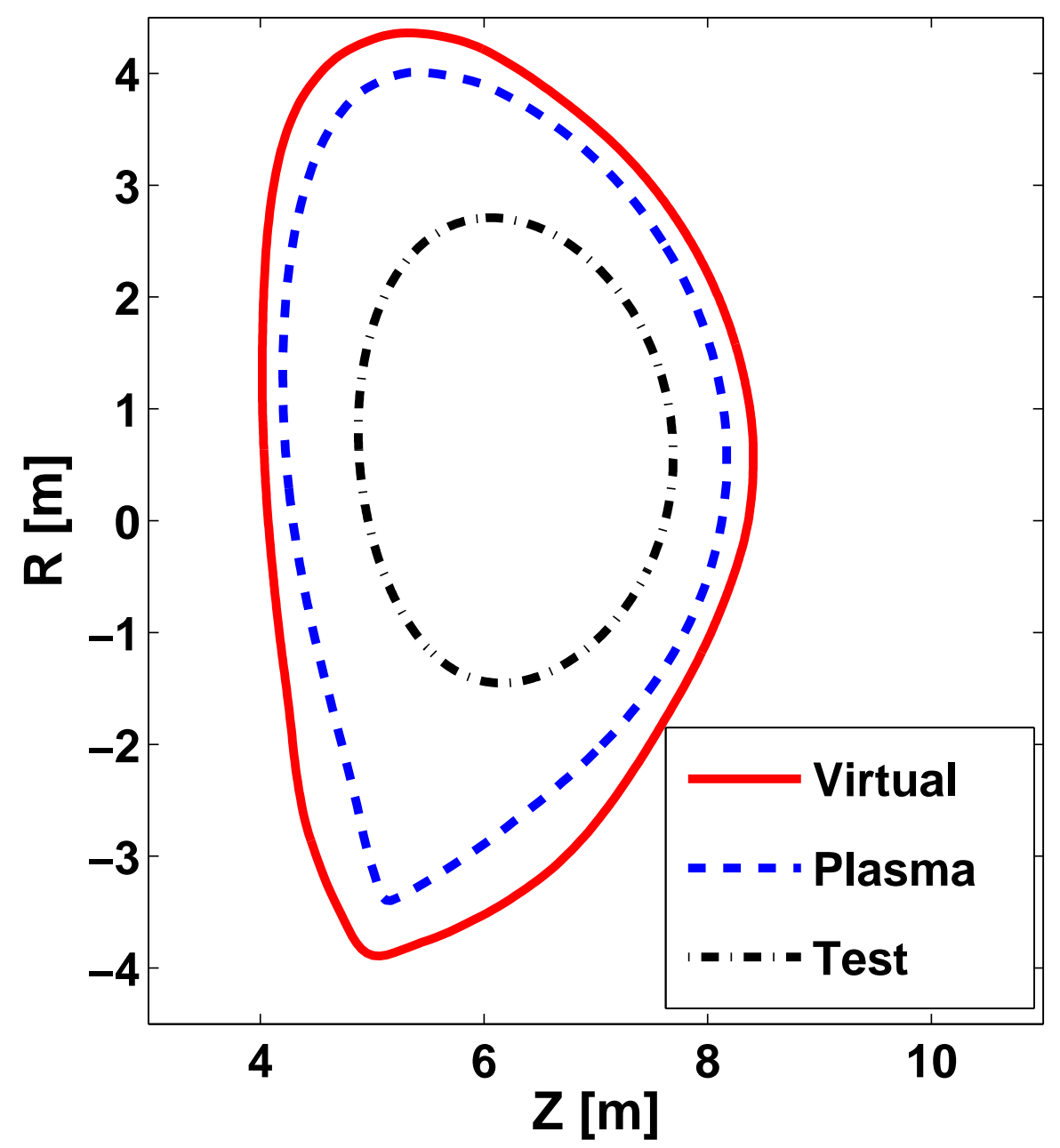

Figure 3: An example of the virtual surface (VS, solid line) where the normal component of the vacuum magnetic field is specified, plotted together with the plasma boundary shape (dashed line) and a test surface (dash-dotted line). Considered here is the 15MA baseline scenario at flat-top, with the $n=1$ vacuum field. 


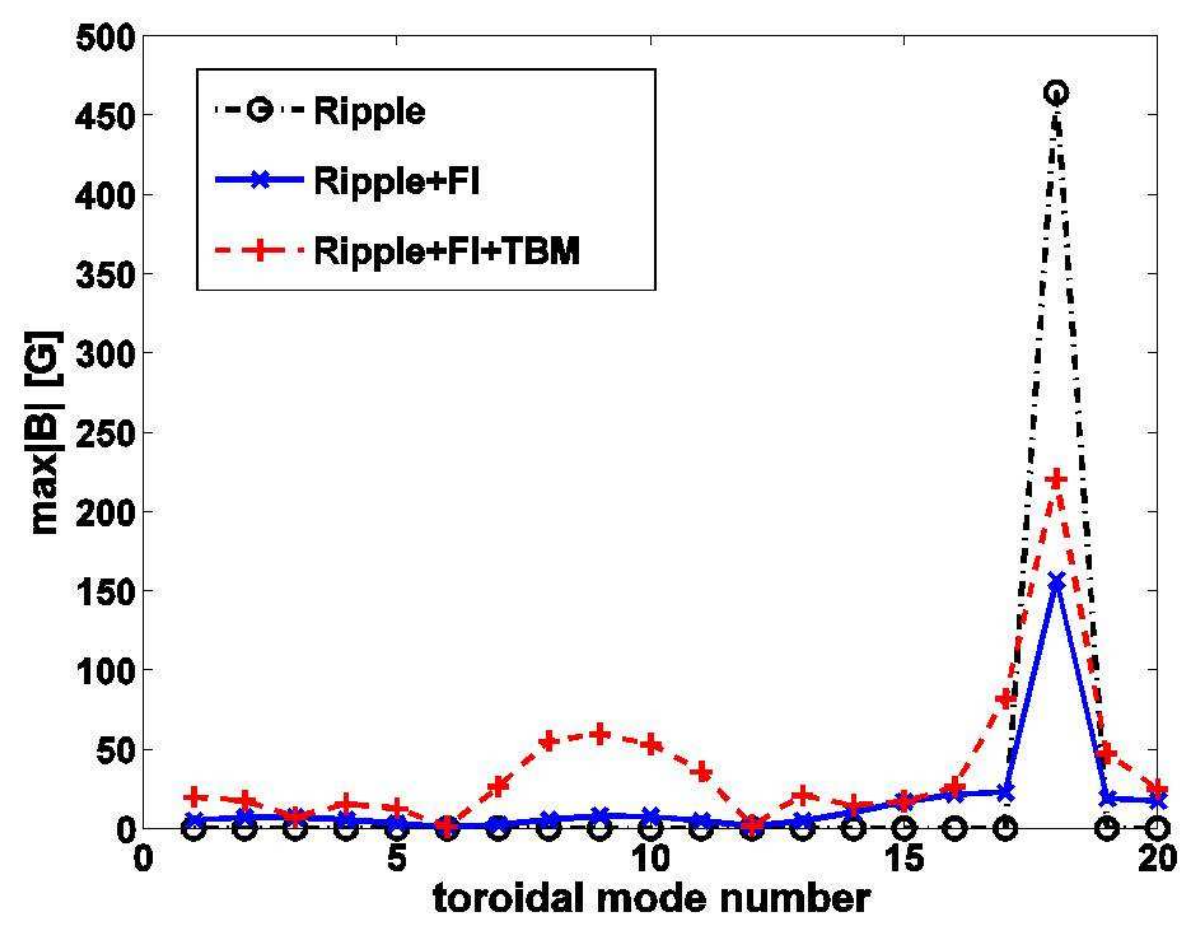

Figure 4: Comparison of the peak amplitude of the vacuum field $|B|$ inside the virtual surface, for toroidal harmonics $n=1-20$, of the ripple field (dash-dotted), the ripple plus the FI field (solid), and of the total field (ripple + FI + TBM, dashed line). The 9MA steady state plasma is considered here.

In this work, mainly three contributions of the external fields (ripples, FIs, TBMs) are provided as the input data. The toroidal Fourier harmonics of these fields are then computed and analysed for each individual $n$-component. In particular, analysis of these input vacuum fields show that the ferritic inserts do compensate, though only partially, the $n=18$ ripple field, as observed in Fig. 4. The peak amplitude of the field inside the VS is compared in the figure. Note that, while compensating the ripple field at $n=18$, FIs also introduce other $n$ components of the vacuum field, though at low level. The largest vacuum fields, with a broad toroidal spectrum, are generated by the TBMs.

\section{Plasma response to 3D fields by FIs and TBMs}

In this Section, we report and analyse the MARS-F plasma response computations for all the four ITER scenarios as described in the previous Section. Before showing the computed plasma response, we discuss two particular issues relevant to the plasma response computations. 


\subsection{Plasma response at high $n$ and computing magnetic field outside vir- tual surface}

The first issue is related to the plasma response for high- $n$ vacuum field components, since both the ripple field and the TBM field contain rather high- $n$ components as shown in Fig. 4.

Using MARS-F, we computed the plasma response to the $n=18$ ripple field, for the 9MA plasma. A comparison of the total field (including the plasma response) with that of the vacuum field, shows almost no difference between these two fields. In order to ensure the numerical convergence, we have included 280 poloidal Fourier harmonics (from $m=-140$ to 140) in computing the plasma response of the $n=18$ field.

We identify two major reasons that the plasma response is weak for high- $n$ harmonics. First, the largest modification, that the plasma response brings to the vacuum field, is for the resonant Fourier harmonics of the radial field. These harmonics are essentially shielded by the plasma response (either ideal response leading to complete shielding, or the resistive plasma response with flow leading to partial shielding). However, for large $n$ field components, the dominant poloidal harmonics, which are normally at low- $m$, are non-resonant and are thus not shielded by the plasma response. For the example shown in Fig. 5, all the harmonics with the $m$ number below 23 are non-resonant. The first resonant harmonic for this $n=18$ radial field is $m / n=$ $24 / 18$, since the $q_{\min }$ value is 1.3248 for this 9MA plasma equilibrium. Since all the $m>23$ Fourier harmonics have at least 3 orders of magnitude lower field, their contribution to the total field, even taking into account the plasma response induced modification, is small. Second, the plasma is normally deeply stable with respect to the high- $n$ kink mode. Therefore, the plasma response induced kink amplification, which is often observed in low- $n$ RMP response modelling $[12,28,10,13]$, does not occur for high- $n$ fields.

In fact, as will be shown later on, the plasma response is already relatively weak for the $n$ numbers above 4 , for all the plasma scenarios considered in this work. Therefore, in most of the work, we shall perform the plasma response computations for $n=1$ to 6 .

Another important issue is how to obtain the total plasma response field which is valid in the whole computational domain. The ESC procedure, as devised in Ref. [21], only ensures that the plasma response is valid inside the VS. What we have realized, however, is that it is possible to rigorously obtain the plasma response in the whole domain even beyond the VS. The key idea here is to first compute and store the perturbed (3D) plasma currents, as the result of the plasma response to the external fields. Next, the magnetic field, produced by the perturbed plasma current, is computed based on a procedure equivalent to the Biot-Savart law. Finally, the plasma current perturbation induced field, and the original vacuum field, which can both be evaluated in the whole space, are combined to obtain the total response field. Examples of the this new procedure are shown below. 


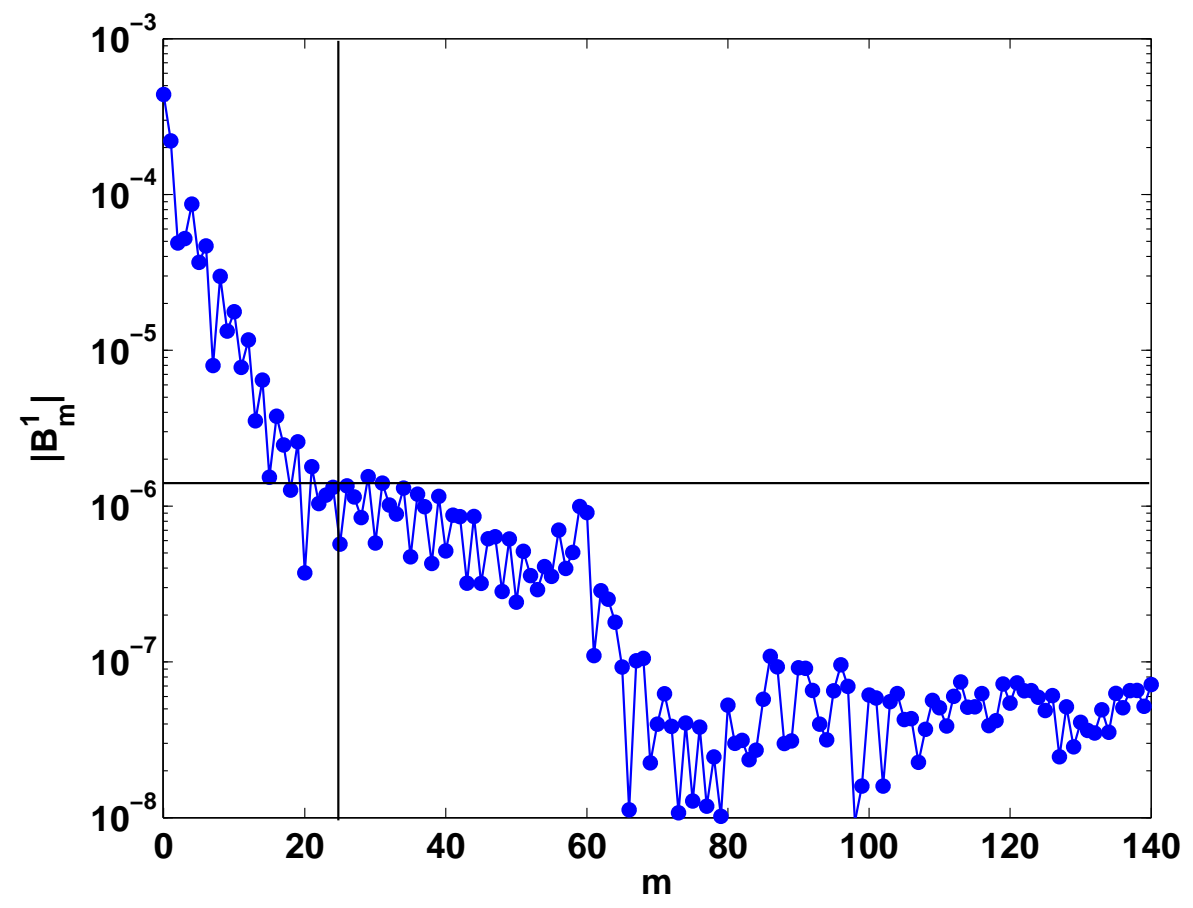

Figure 5: The logarithmic plot of the $n=18$ vacuum radial field amplitude versus the poloidal harmonic number $m$, for the combined field from ripples, FIs and TBMs of the 9MA case.

\subsection{Plasma response for four ITER scenarios}

The plasma response has been computed for all four ITER scenarios, with six plasma equilibria in total as listed in Tab. 1. For each equilibrium, the response is computed for each individual $n=1-6$ field component. For each equilibrium and each $n$, we separately compute the plasma response to the ripple $+\mathrm{FI}$ and ripple $+\mathrm{FI}+\mathrm{TBM}$ fields. In addition, the plasma response to the ELM control coil currents is also computed for the 15MA baseline scenario and the 9MA steady state scenario, again for each individual $n=1-6$. For the RMP fields, the corresponding (optimal) coil configurations are taken from Ref. [32]. Some of these response field data have been used for further investigation of the fast ion losses in ITER [5].

As an example, figure 6 plots the plasma response field (only the $B_{R}$ component along $R$ at $Z=-0.03 \mathrm{~m}$ is shown) for all $n=1-6$ components, for the 9MA steady state equilibrium at FT. The ripple, FI and TBM contributions are all included. Addition of the TBM contribution normally results in several times larger field than the FI field. It's also interesting to note that the plasma response is not very strong (compared to the corresponding vacuum field) when all fields are included. It turns out that the plasma response leads to larger modification of the vacuum field, when the TBM contribution is absent. This shows that the poloidal spectrum of the applied vacuum field can significantly affect the plasma response. It is also evident that the pure plasma response (i.e. the fields produced by the perturbed plasma response currents, shown in red) is already small, compared to the applied vacuum field (shown in blue), for the $n=4$-component. 

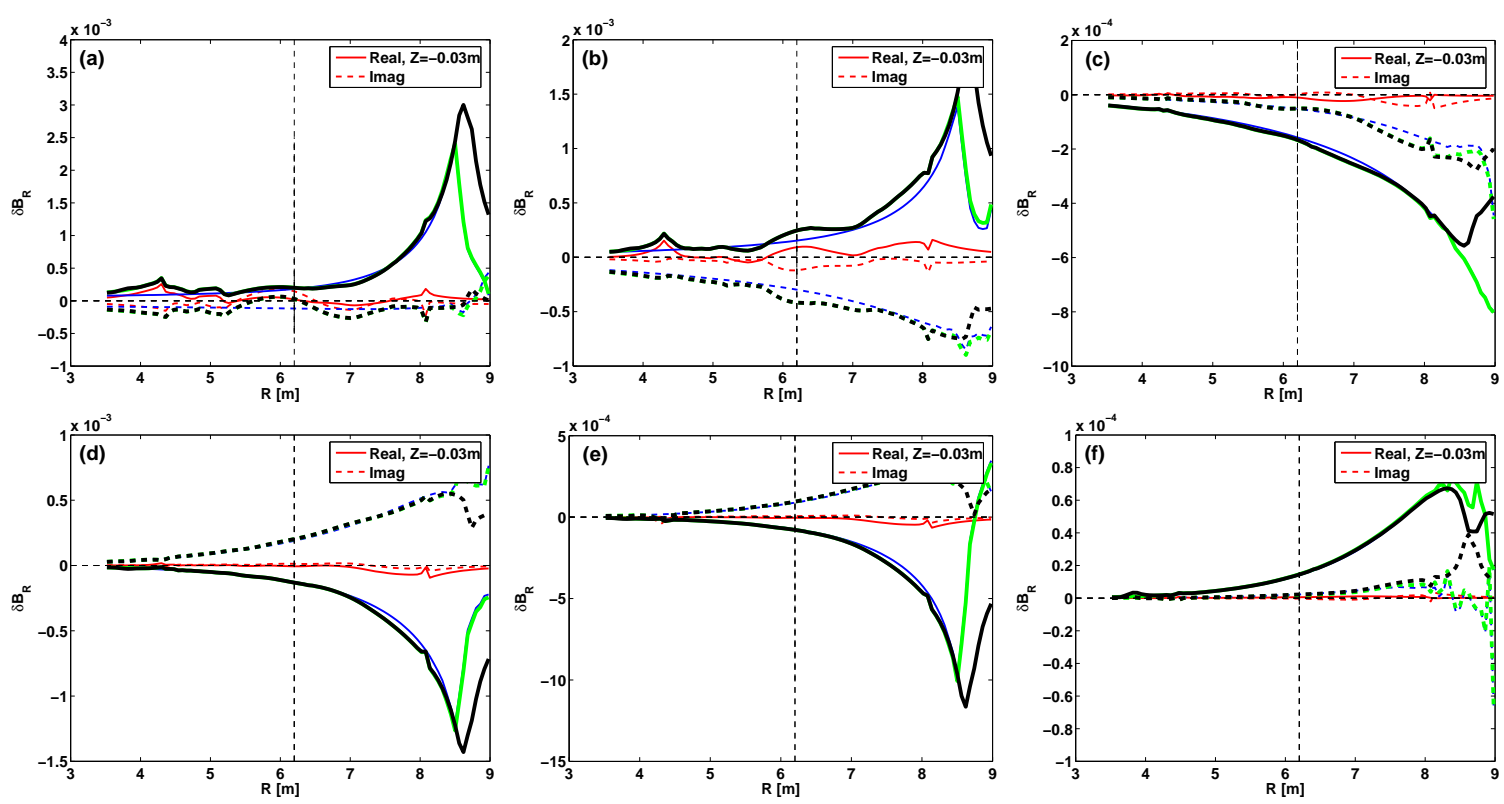

Figure 6: The computed $B_{R}$ field (in Tesla), both real (solid) and imaginary (dashed) parts, along the major radius at the vertical position $Z=-0.03 \mathrm{~m}$, for the 9MA steady state scenario at the flat-top phase and including the field contributions from the ripple, the FI and TBM. Each of the sub-plots (a-f), corresponding to $n=1-6$, respectively, shows (i) the vacuum field (blue), (ii) the response field produced by the perturbed plasma current (red), (iii) the directly computed total field valid within the virtual surface (black, in the major radius range between $4 \mathrm{~m}$ and $8.4 \mathrm{~m}$ in this plot), and (iv) the total field valid everywhere, by combing fields (i) and (ii). 

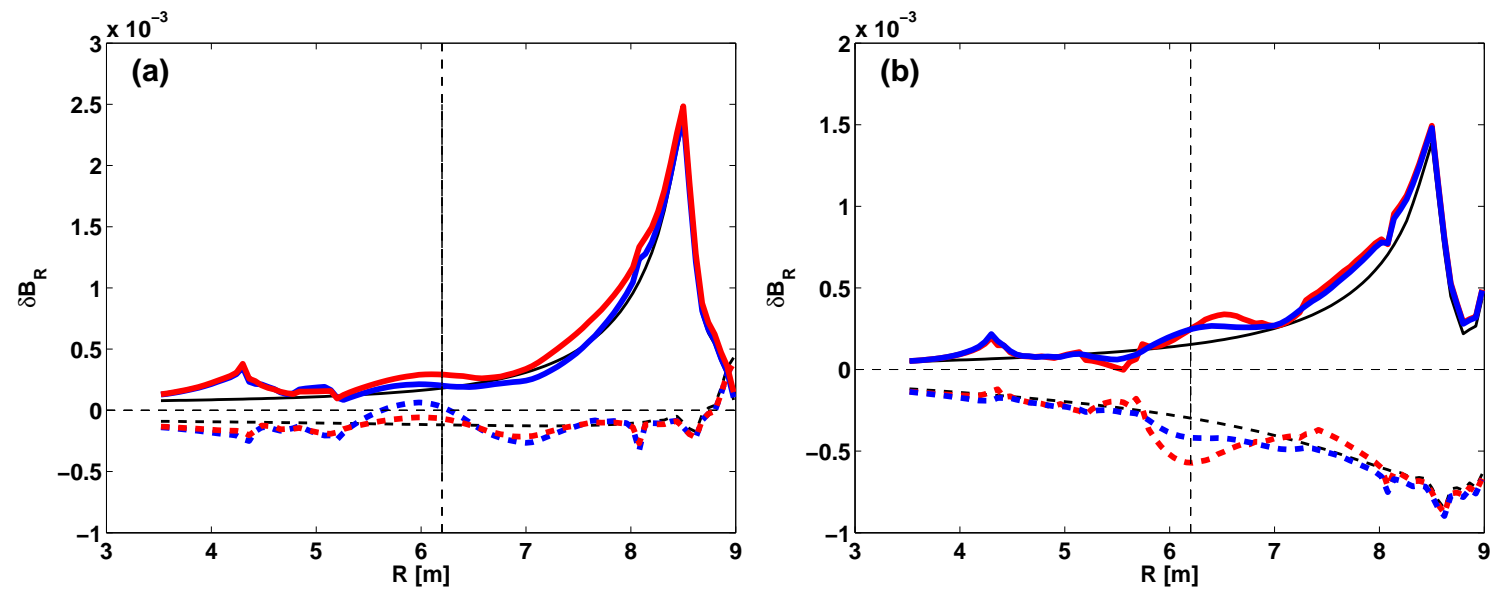

Figure 7: The computed $B_{R}$ field (in Tesla), both real (solid) and imaginary (dashed) parts, along the major radius at the vertical position $Z=-0.03 \mathrm{~m}$, for the $9 \mathrm{MA}$ steady state scenario at the flat-top phase and including (a) the $n=1$, and (b) $n=2$, field contributions from the ripple, the FIs, TBMs, as well as (the low- $n$ side-bands of) the ELM control coils. Compared are (i) the vacuum field (black), (ii) the total response field assuming the fluid model (blue), and (iii) the total response field assuming the drift kinetic model (red).

For the 9MA equilibrium, which has high beta, we have also tested whether the drift kinetic effects from thermal particles can significantly modify the low- $n$ plasma response. The kinetic effects include the resonances between the mode and the drift motions of bulk plasma particles species, including the toroidal precession of thermal ions and electrons, the bounce (transit) motion of trapped (passing) thermal ions. The comparison, shown in Fig. 7, indicates that the drift kinetic modification is moderate for this ITER equilibrium, for the $n=1$ and $n=2$ plasma response.

Figure 8 compares the $n=1$ plasma response for all 6 equilibria considered in this work. The plasma response appears particularly strong for the 7.5MA half-field Helium plasma scenario. This is associated with a strong core kink amplification effect by the plasma $[12,28]$.

\subsection{Sensitivity of plasma response to flow variation}

Due to the well known uncertainty in the transport prediction of the toroidal flow speed for ITER (one example is shown in Fig. 1(d)), it is important to verify whether the MARS-F computed plasma response is sensitive to the equilibrium flow. We choose the 15MA FT plasma for this study. We consider the $n=1$ ripple plus FI fields only, since the plasma response yields relatively larger modification to the vacuum field, in the absence of the TBM field.

Figure 9 shows the MARS-F computed total response field while artificially scanning the whole flow profile, obtained by the JINTRAC modelling assuming the Prandtl number of 

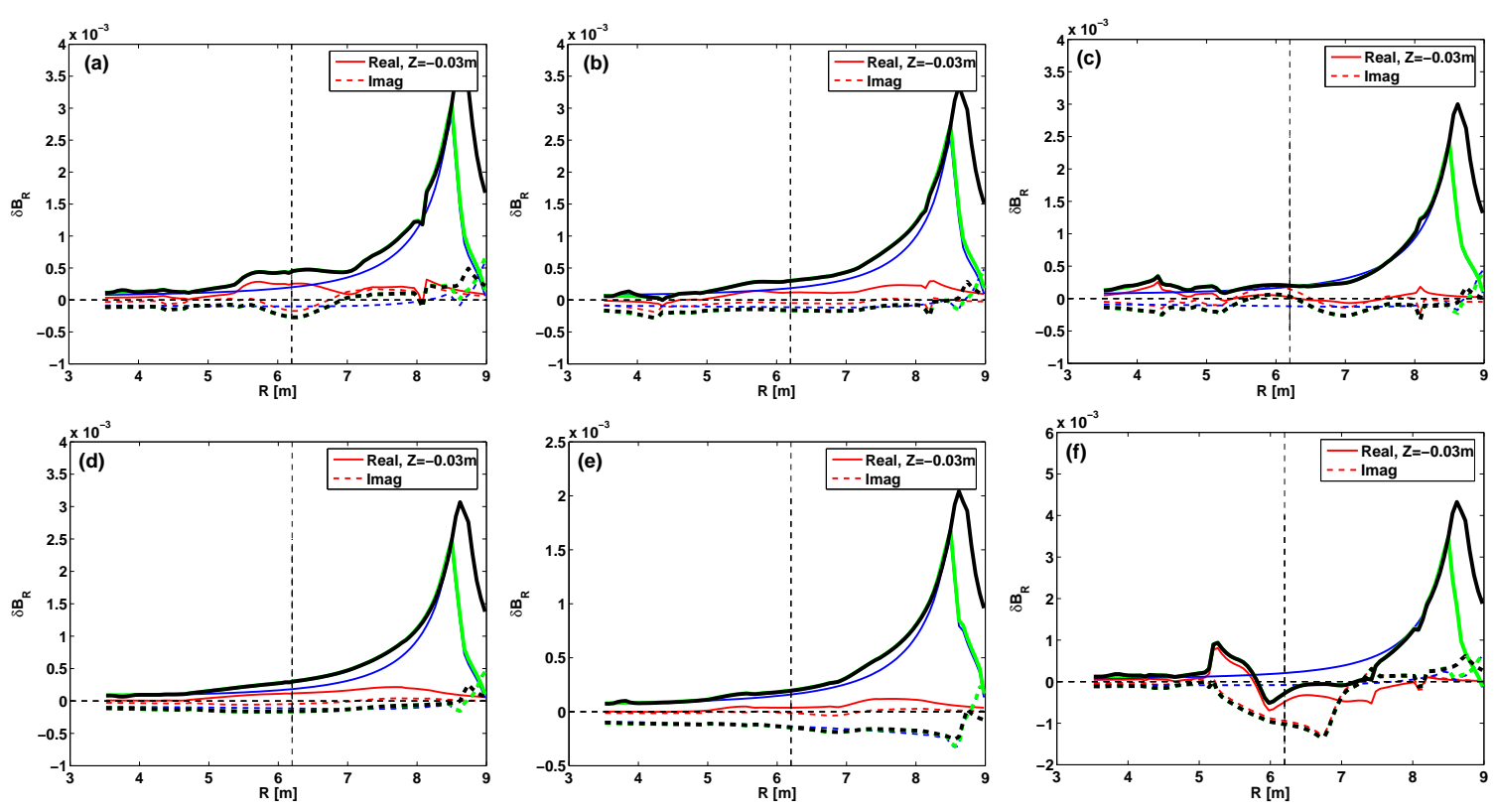

Figure 8: The computed $n=1 B_{R}$ field (in Tesla), contributed by the ripple, the FI and TBM fields, both real (solid) and imaginary (dashed) parts, plotted along the major radius at the vertical position $Z=-0.03 \mathrm{~m}$. Each of the sub-plots (a-f), corresponding to (a) the 15MA baseline scenario at flat-top, (b) the 12.5MA hybrid scenario at flat-top, (c) the 9MA steady state scenario at flat-top, (d) the 15MA baseline scenario at ramp-up, (e) the 12.5MA hybrid scenario at ramp-up, and (f) the 7.5MA half-field Helium scenario at flat-top, respectively, shows (i) the vacuum field (blue), (ii) the response field produced by the perturbed plasma current (red), (iii) the directly computed total field valid within the virtual surface (black, in the major radius range between $4 \mathrm{~m}$ and $8.4 \mathrm{~m}$ in this plot), and (iv) the total field valid everywhere, by combing fields (i) and (ii). 

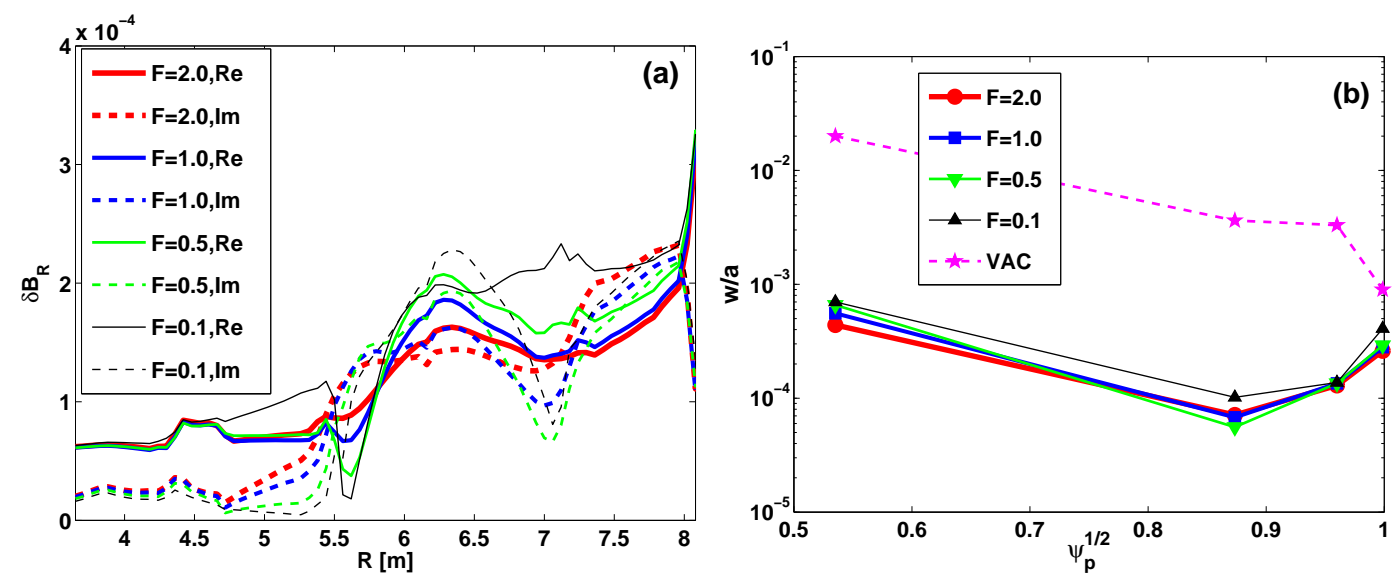

Figure 9: The computed (a) real (solid) and imaginary (dashed) parts of the total $n=1$ response field $\delta B_{R}$, plotted along the major radius $R$ across the mid-plane $Z=0$, and (b) magnetic islands width at rational surfaces, while varying a scaling factor $F$ for the toroidal rotation amplitude. The whole radial profile of the toroidal rotation frequency, as shown in Fig. 1(d) for the Prandtl number of 0.75 , is scaled by the factor $F$. The islands produced by the vacuum field is also plotted in (b). Considered is the 15MA scenario at flat-top, with the inclusion of both the ripple and the FI fields.

0.75 , by a factor $F$, which varies between 0.1 and 2 . The total response field does not significantly change when $F$ varies within a factor of 2 along both ends. This also indicates the difference between the two rotation profiles shown in Fig. 1(d) should not strongly affect the computed plasma response. Indeed the MARS-F computations confirm that the plasma response is almost identical using these two flow profiles from Fig. 1(d).

However, the plasma response does significantly change, if the flow speed is reduced by one order of magnitude, as shown in Fig. 9. Similar observations have been made by previous studies $[12,10]$.

\subsection{Evaluation of the Chirikov parameter}

Associated with the resonant 3D field perturbations are the magnetic islands and the Chirikov parameter. These islands are formed as a result of the forced reconnection. The MARS-F resistive plasma response model enables us to compare the island width (and consequently the Chirikov parameter) with that of the vacuum approximation.

The MARS-F computed radial field perturbation is decomposed in Fourier harmonics, in a PEST-like straight field line coordinate system

$$
Q=\left(\frac{\mathbf{b} \cdot \nabla \psi_{p}}{\mathbf{B}_{\mathrm{eq}} \cdot \nabla \phi}\right)_{m n},
$$

where $Q$ denotes the $(m, n)$-th Fourier harmonic of the perturbed radial magnetic field $\mathbf{b} . \psi_{p}$ 
is the equilibrium poloidal flux function, which also labels the radial coordinate $r$, with $r \equiv$ $\sqrt{\left(\psi_{0}-\psi_{p}\right) /\left(\psi_{0}-0\right)}$. Note that here we have assumed that the equilibrium poloidal flux is $\psi_{0}$ on the magnetic axis, and vanishes at the plasma edge. $\mathbf{B}_{\mathrm{eq}}$ denotes the equilibrium magnetic field, and $\phi$ is the geometric toroidal angle.

As shown in Appendix, the width of the magnetic island, located at the rational surface $q=$ $m / n$, can be evaluated, in general toroidal geometry, as

$$
w=\frac{\Delta r}{a}=4 \sqrt{\left|\frac{Q}{2 \psi_{0} n S}\right|},
$$

where $S \equiv(r / q) d q / d r$ is the magnetic shear, evaluated at the same rational surface.

Assuming two neighbouring islands, of width $w_{1}$ and $w_{2}$, are located at the minor radii of $r_{1}$ and $r_{2}$, respectively, the Chirikov parameter is conventionally defined as

$$
\sigma=\frac{\left(w_{2}+w_{1}\right) / 2}{\left|r_{2}-r_{1}\right|}
$$

In the following, we shall plot the Chirikov parameter for the ITER 15MA scenario, based on the computed plasma response. A similar investigation has been carried out for the 9MA case but not shown here. Before showing the results, we point out that the Chirikov parameter only provides an estimate of the field line stochasticity, induced by the islands overlapping. A more accurate knowledge is obtained by direct field line tracing (Poincare plot) using the computed plasma response field.

We have compared the computed Chirikov parameter under the vacuum field (ripple plus FI fields) assumption, between the current ramp-up phase and the flat-top phase, for the 15MA baseline scenario and for each of the $n=1-6$ toroidal components. As expected, the Chirikov parameter is generally larger, for all $n$ 's, during the RU phase. Each single $n$ component does not yield significant islands overlapping even near the plasma edge - the Chirikov parameter is always below 1 .

As one example, figure 10 compares the Chirikov parameter without (blue) and with (red) the plasma response, for each $n$-component of the 15MA FT case with the applied ripple plus the FI fields. Except for the $n=6$ case, the plasma response generally reduces the magnetic island width compared to the vacuum island, and hence the Chirikov parameter as well. The reduction is significant towards the plasma core, but generally moderate near the plasma edge, due to both higher plasma resistivity (lower thermal electron temperature) and slower plasma flow in the edge region. The amplification of the magnetic islands by the plasma response, shown here for the $n=6$-component, is also plausible, as has already been noted in previously studies $[33,34]$.

Combining all $n$-components yields Chirikov parameter locally exceeding 1 , as shown in Fig. 11. This is largely because more islands, of different helicities, now co-exist at very close distances. The Chirikov parameter, as a function of the plasma minor radius, thus shows a rather irregular behaviour when combining together all islands with different $n$-numbers. This 

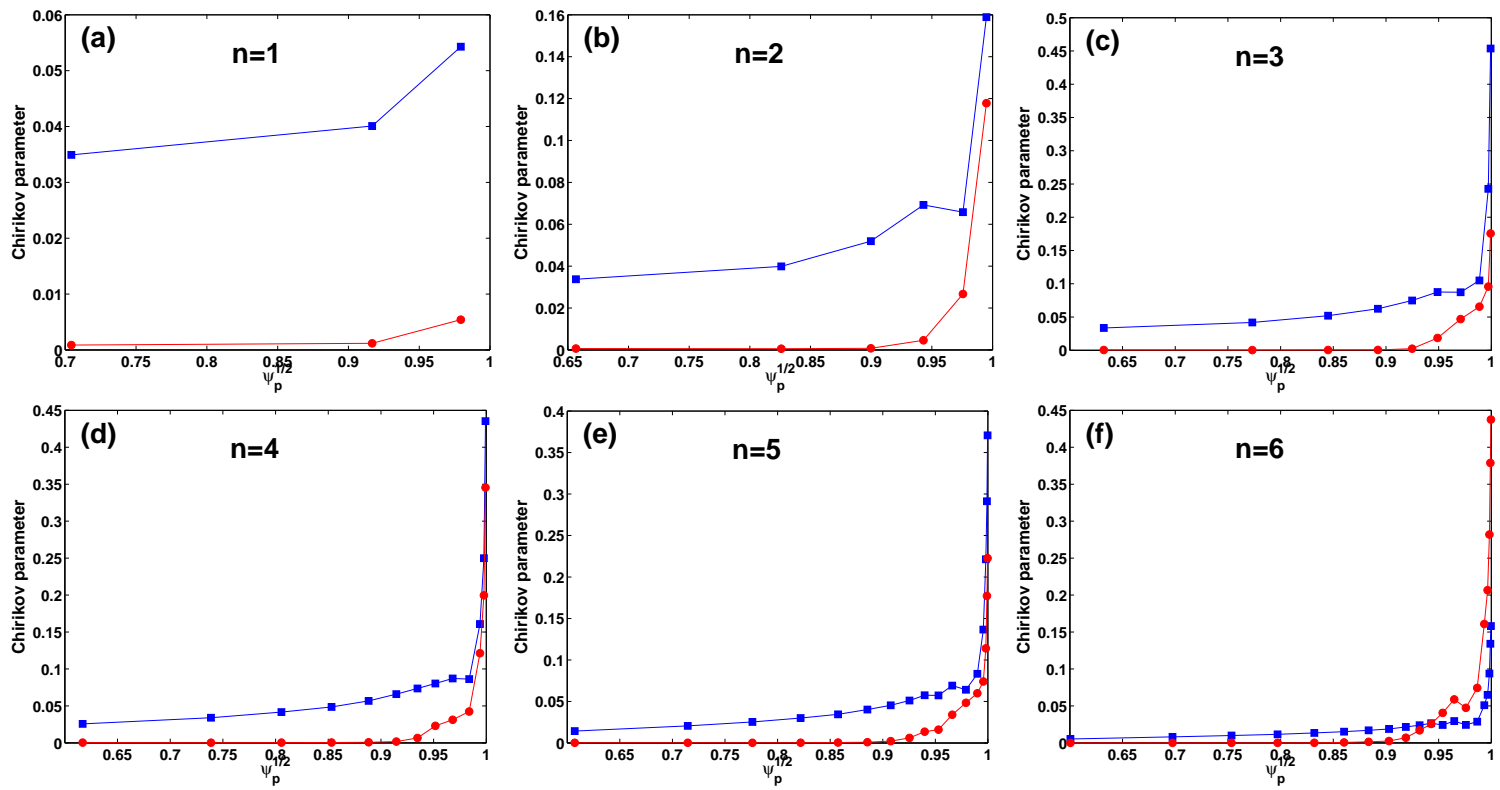

Figure 10: Comparison of the Chirikov parameters, corresponding to each individual $n=1-6$ ripple + FI vacuum (blue) and total response (red) field, for the 15MA scenario at the flat-top phase.

complicates the judgement on the field line stochastization near the plasma edge region. A better definition rather than simply the Chirikov parameter, such as that defined in Ref. [32], can be more useful. Eventually the best way of judging the field line stochasticity, is probably still the Poincare field line tracing plot. Nevertheless, Fig. 11 still quantitatively shows clear reduction of the Chirikov parameter by the plasma response.

We also find that, for a given $n$ ( $n=3$ and $n=4$ for ITER), the largest field perturbation comes from the ELM control coils (the RMP fields). On the other hand, the symmetry of the ELM coil distribution along the toroidal angle in ITER normally generates narrow band toroidal spectrum of the 3D field perturbation, compared to the rather broad spectrum (for $n$ up to 20) generated by the ripples, FIs, and particularly the ITER TBMs.

\section{Error field correction using EFCC}

\subsection{EFCC specification}

In this work, we consider using the ITER error field correction coils (EFCC) to correct the 3D fields produced by the FIs and TBMs. The correction takes into account the plasma response as computed by MARS-F, following various EFC optimization criteria.

The EFCC design, shown in Fig. 12, is taken from Ref. [35]. Each row consists of 6 coils, 

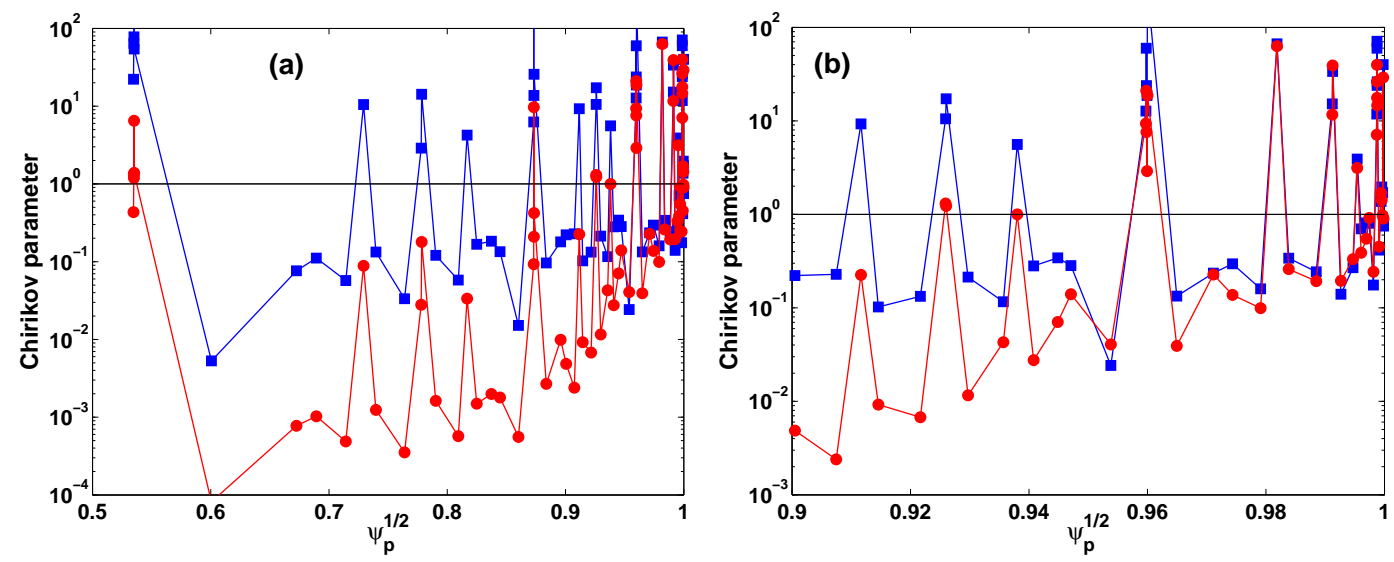

Figure 11: Comparison of the Chirikov parameters, corresponding to all $n=1-6$ vacuum (blue) and total response (red) ripple+FI fields combined together, for the 15MA scenario at the flat-top phase, (a) in the whole plasma region, and (b) near the plasma edge.

covering 60 degrees along the toroidal angle for the top and bottom rows, and 36 degrees for the mid-plane row. The upper limit of the coil current is $10 \mathrm{kA}$, with 32 turns designed for the top and bottom rows, and 20 turns for the mid-plane row of EFCC.

Here we shall consider the correction of the $n=1$ field component, by three rows of the EFCC. This is motivated by the fact that normally the $n=1$ field component leads to the most severe consequences for the mode locking. We shall consider the EFC for the 15MA scenario at the flat-top phase. Two studies, with and without the TBM contribution to the EF, shall be carried out. Here by the EF we specifically refer to the combined fields from the ripple, the FIs, and/or the TBMs.

\subsection{Criteria for EFC optimization}

We shall consider various optimization criteria, following a similar study that has been carried out for the MAST plasmas [21]. In particular, according to Criteria A, we choose the EFCC currents such that the $m / n=2 / 1$ resonant component of the total field (EF + EFCC) vanishes at the $q=2$ surface. This choice is motivated by the fact that the mode locking observed in experiments is often associated with the $2 / 1$ tearing mode. Two possibilities are considered: either the full cancellation of the vacuum EF only, by the vacuum EFCC (further referred to as Criterion $\mathrm{AV}$ ), or that of the full field including the linear resistive plasma response to both $\mathrm{EF}$ and EFCC (further referred to as AP).

With Criterion $\mathrm{B}$, we minimize the net resonant electromagnetic torque (the $\mathbf{j} \times \mathbf{b}$ torque) acting on the whole plasma column, due to the plasma response to both the EF and the EFCC field. This is again motivated by the mode locking physics.

The third family of criteria, Criteria $\mathrm{C}$, is designed to minimize various aspects of the 3D 


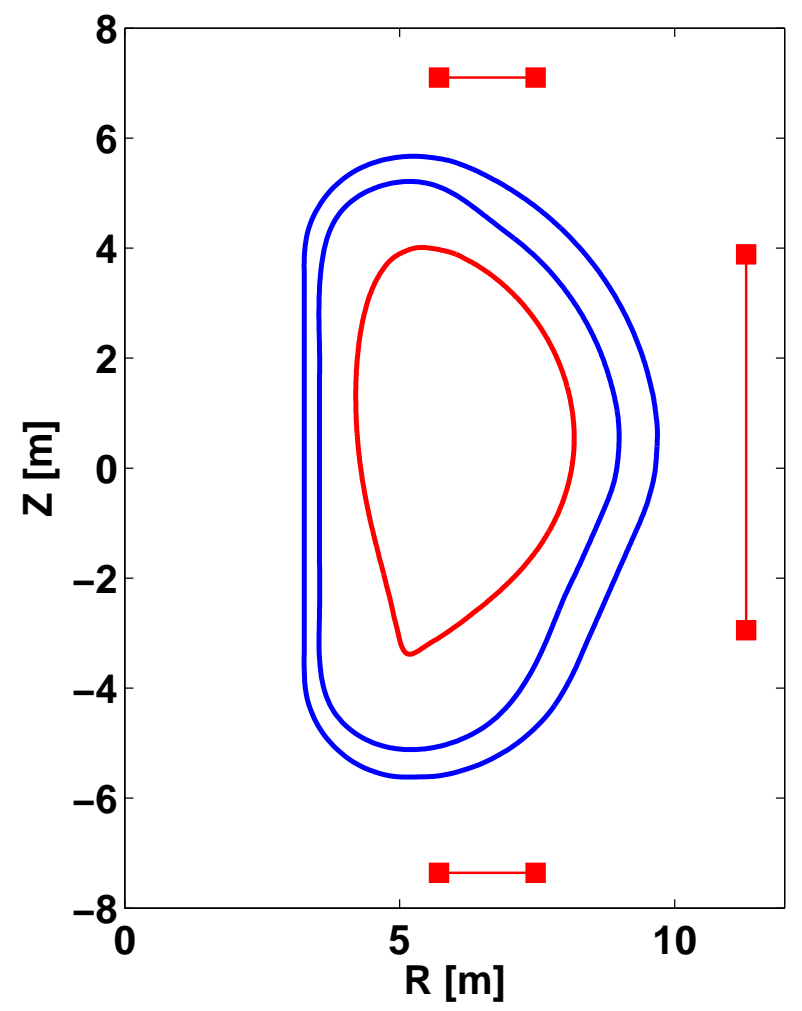

Figure 12: Location of the top, mid-plane, and bottom rows of the EFCC in ITER, plotted together with the plasma boundary shape (red) and the double vacuum vessel model (blue). 
corrugation of the plasma surface, as a result of the plasma response to 3D EF. This includes the overall peak displacement of the plasma surface (further referred to as Criterion CA), the averaged value of the surface displacement (AE), the low field side mid-plane (CI) as well as the maximal displacement near the equilibrium X-point (CX). This family of criteria, initially designed for correcting the EF in MAST plasmas [21], may also be of practical usefulness in ITER (e.g. to minimize the peak amplitude of the plasma displacement thus avoiding the plasma locally touching the first wall).

The actuators for the EFC optimization are obviously the three rows of EFCC currents. Assuming that the $n=1$ currents flowing in the upper, lower and middle rows are specified as $I^{U} \exp \left(i \Phi^{U}\right), I^{L} \exp \left(i \Phi^{L}\right)$, and $I^{M} \exp \left(i \Phi^{M}\right)$, respectively, we end up with a generally sixdimensional optimization problem in the real space $\left(I^{U}, I^{L}, I^{M}, \Phi^{U}, \Phi^{L}, \Phi^{M}\right)$. In order to simplify the problem, we shall fix certain parameters, and carry out most of the optimization in two-dimensional sub-spaces. Such approach not only simplifies the optimization procedure, but also allows easy illustration of the robustness of the obtained optima. Even though a systematic investigation has been performed, we shall report below only sample results illustrating the key results.

\subsection{EFC optimization results}

As the first study, we choose three typical cases of specifying the EFCC current amplitude: (i) $I^{U}=I^{L}=I^{M}$, (ii) $2 I^{U}=2 I^{L}=I^{M}$, (iii) $I^{U}=I^{L}=2 I^{M}$. For each case, we assume the same toroidal phase for the upper and lower rows of coils, i.e. $\Phi^{U}=\Phi^{L}=\Phi$, and independently vary the two phase parameters $\left(\Phi, \Phi^{M}\right)$. The assumption of $\Phi^{U}=\Phi^{L}=\Phi$ is not unique but representative. In fact we have also made the optimization assuming $\Phi^{U}=-\Phi^{L}=\Phi$, but find that the optimal results are not sensitive to this.

We adopt the following optimization procedure. First, we run the MARS-F code to compute the plasma response fields (or the vacuum field for the vacuum field based Criterion AV) for each individual row of coils, assuming a unit current amplitude and zero toroidal phase. Next, we perform superposition of the computed fields, by linearly scaling each of the fields by the coil currents in the corresponding rows. The superposition is always valid for linear plasma response. The EFC optimization has been carried out without or with the TBM field.

One example, based on the vacuum field correction Criterion AV, is shown in Fig. 13. Here only the ripple and FI contributions are included. We fix the coil current amplitude at $I^{U}=$ $I^{L}=I^{M}=I=10 \mathrm{kAt}$, and varying the coil's phasing in the $2 \mathrm{D}$ domain of $\left(\Phi^{U}=\Phi^{L}=\Phi, \Phi^{M}\right)$. the optimal point, $\Phi=90^{\circ}$ and $\Phi^{M}=100^{\circ}$, is shown by the " + " symbol in the figure. Note that, even though the optimal (corresponding to minimal $b_{2 / 1}^{1}$ amplitude) point is relatively robust, there is also a global maximal point at $\Phi=90^{\circ}$ and $\Phi^{M}=280^{\circ}$ that corresponds to the worst correction of the $n=1$ vacuum island.

Taking into account the plasma response, however, significantly shifts the optimal point as shown by Fig. 14. The optimal coil phasing now becomes $\left(\Phi^{U}=\Phi^{L}=340^{\circ}, \Phi^{M}=330^{\circ}\right)$. Note also the significantly reduced field amplitude, compared to the vacuum field shown in 


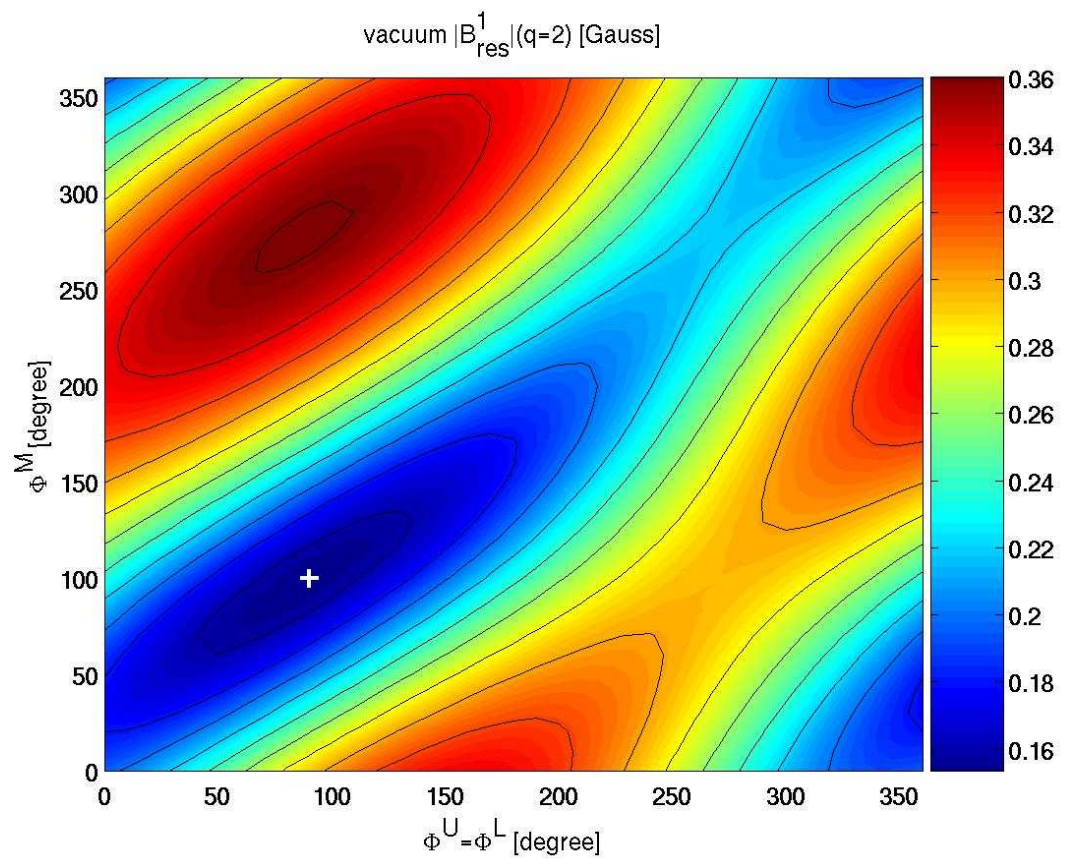

Figure 13: The amplitude of the $m / n=2 / 1$ vacuum radial field at the $q=2$ rational surface with varying toroidal coil phasing for EFCC. The vacuum field is the combination of the ripple+FI fields, and the EFCC field assuming $I^{U}=I^{L}=I^{M}=I=10 \mathrm{kAt}$.

Fig. 13.

The next example also includes the TBM field contribution. The results, following optimization criterion B, is reported in Tables 2, for various choices of combination for the coil current amplitudes. At each combination, the optimal EFCC current phasing is presented. Overall, it is interesting to note that, with the inclusion the TBM contribution, the optimal phasing for the middle row EFCC remains relatively fixed at around $\Phi^{M}=300^{\circ}$, according to all but the vacuum criteria.

As an example, one optimum point from Tab. 2, with $2 I^{U}=2 I^{L}=I^{M}=I=18 \mathrm{kAt}, \Phi=120^{\circ}$ and $\Phi^{M}=300^{\circ}$, is shown by the "+" symbol in Fig. 15 . The negative value of the torque indicates the net $\mathbf{j} \times \mathbf{b}$ acts to brake the plasma flow.

Next, we fix the EFCC current phasing and optimize the current amplitude. We choose two cases of specifying the EFCC current phase: (i) $\Phi^{U}=\Phi^{L}=\Phi^{M}=\Phi$, and (ii) $\Phi^{U}=\Phi^{L}=$ $0, I^{M}=\Phi$. For each case, we assume the same current amplitude for the upper and lower rows of coils, i.e. $I^{U}=I^{L}=I$, and independently vary the two amplitudes $\left(I, I^{M}\right)$. We adopt the similar procedure to that described for the coil phasing optimization.

Even though the optimum varies depending on the applied 3D field configuration (without or with TBM), and on the chosen criterion for optimization, an overall conclusion is that the dominant correction comes from the middle row EFCC for the ripple, FI and/or TBM fields. This is also evident from one example shown in Fig. 16, by the fact that the optimum point 


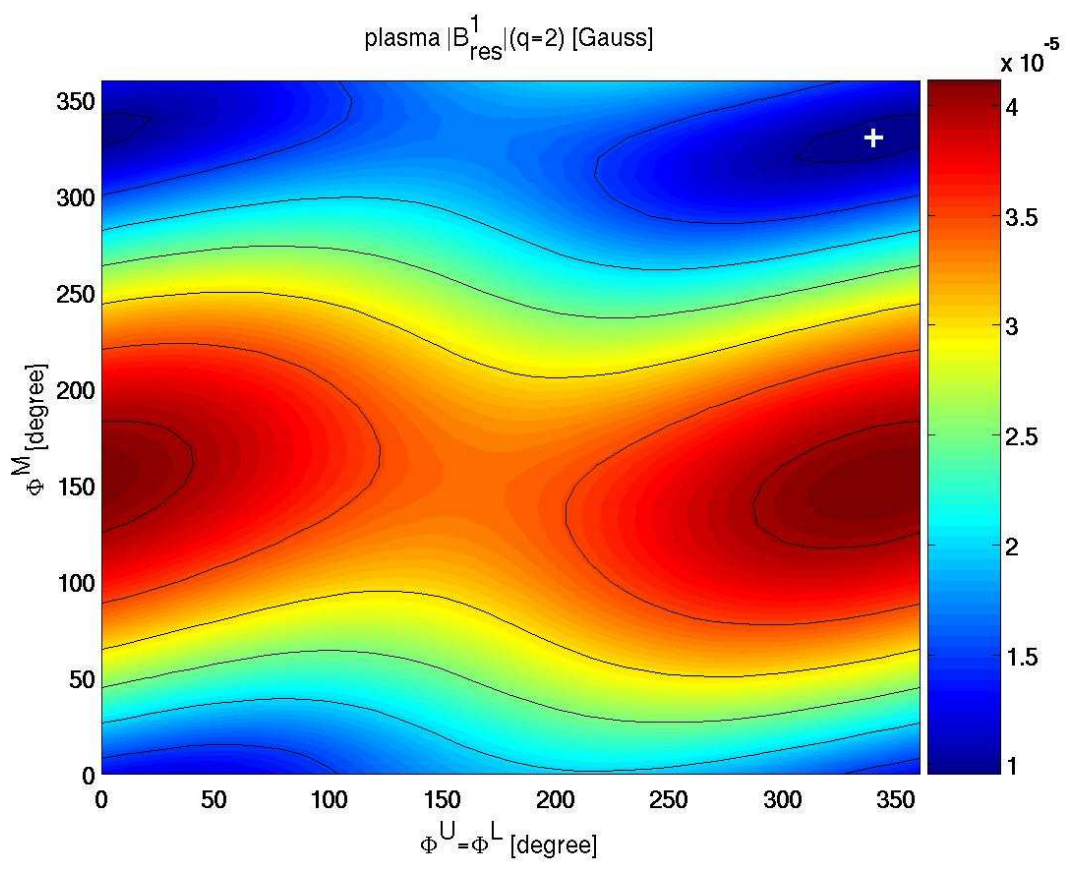

Figure 14: The amplitude of the $m / n=2 / 1$ total response radial field at the $q=2$ rational surface with varying toroidal coil phasing for EFCC. The field is the combination of the plasma response to the ripple+FI fields, and to the EFCC field assuming $I^{U}=I^{L}=I^{M}=I=10 \mathrm{kAt}$.

Table 2: Optimal EFCC current phasing (in degrees) for correcting ripple+FI+TBM fields following the plasma response based Criterion B. Here $\Phi^{U}=\Phi^{L}=\Phi$. $T$ is the minimal net $\mathbf{j} \times \mathbf{b}$ torque for each choice of the EFCC current amplitude.

\begin{tabular}{|r||r|c|c||r|r|r||r|r|r|}
\hline \multicolumn{1}{|c||}{} & \multicolumn{3}{c||}{$I^{U}=I^{L}=I^{M}=I$} & \multicolumn{2}{c||}{$2 I^{U}=2 I^{L}=I^{M}=I$} & \multicolumn{3}{c|}{$I^{U}=I^{L}=2 I^{M}=I$} \\
\hline I(kAt) & $\Phi$ & $\Phi^{M}$ & $T(\mathrm{Nm})$ & $\Phi$ & $\Phi^{M}$ & $T(\mathrm{Nm})$ & $\Phi$ & $\Phi^{M}$ & $T(\mathrm{Nm})$ \\
\hline 2 & 140 & 300 & -2.6760 & 140 & 300 & -2.7866 & 140 & 300 & -2.9474 \\
4 & 140 & 300 & -1.9864 & 140 & 300 & -2.1783 & 140 & 300 & -2.4615 \\
6 & 140 & 300 & -1.4115 & 140 & 300 & -1.6550 & 140 & 300 & -2.0225 \\
8 & 140 & 300 & -0.9512 & 140 & 300 & -1.2169 & 140 & 300 & -1.6304 \\
10 & 130 & 300 & -0.6029 & 130 & 300 & -0.8626 & 140 & 300 & -1.2851 \\
12 & 130 & 300 & -0.3674 & 130 & 300 & -0.5925 & 140 & 300 & -0.9866 \\
14 & 130 & 300 & -0.2453 & 130 & 300 & -0.4069 & 140 & 300 & -0.7351 \\
16 & 110 & 310 & -0.2206 & 120 & 300 & -0.3049 & 140 & 300 & -0.5303 \\
18 & 90 & 310 & -0.2425 & 120 & 300 & -0.2822 & 130 & 300 & -0.3707 \\
20 & 70 & 320 & -0.2625 & 80 & 310 & -0.3139 & 130 & 300 & -0.2563 \\
22 & 60 & 320 & -0.2939 & 60 & 310 & -0.3474 & 130 & 300 & -0.1881 \\
24 & 50 & 320 & -0.3388 & 40 & 310 & -0.4016 & 120 & 310 & -0.1532 \\
26 & 40 & 320 & -0.3930 & 30 & 310 & -0.4764 & 110 & 320 & -0.1410 \\
28 & 30 & 320 & -0.4597 & 340 & 300 & -0.5573 & 110 & 320 & -0.1465 \\
30 & 30 & 320 & -0.5275 & 340 & 300 & -0.6539 & 100 & 330 & -0.1394 \\
\hline
\end{tabular}




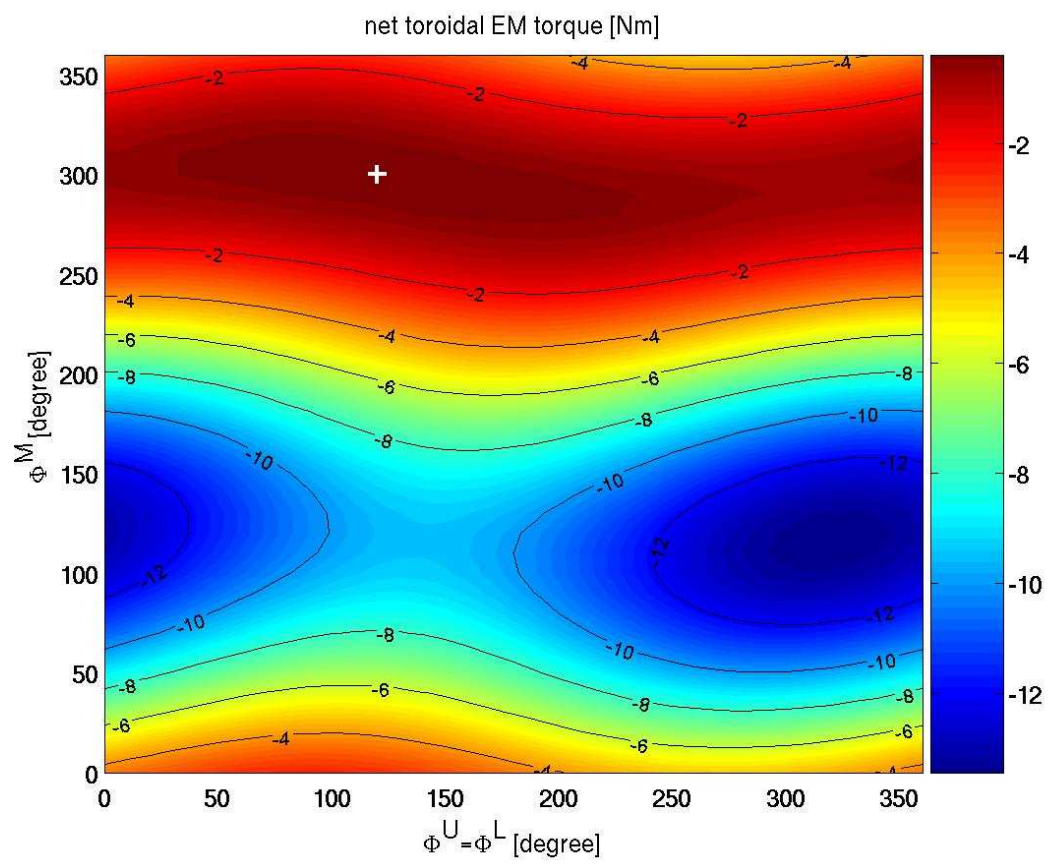

Figure 15: The computed net $\mathbf{j} \times \mathbf{b}$ torque acting on the whole plasma column, with varying toroidal coil phasing for EFCC. The torque occurs due to the plasma response to the combination of the ripple+FI+TBM fields, and the EFCC field assuming $2 I^{U}=2 I^{L}=I^{M}=I=18 \mathrm{kAt}$.

is much more sensitive to the middle row coil current, than the top and bottom rows EFCC currents.

\section{Flow damping due to FI and TBM fields}

Here, we again choose the 15MA baseline scenario at the flat-top phase. We run the MARS-Q [18] code to model the time evolution of the toroidal flow, assuming the presence of (i) the ripple + FI fields, (ii) the ripple + FI + TBM fields, and (iii) the ripple + FI + TBM + RMP fields. As in the case of the EFC study (and following the same motivation), we consider only the $n=1$ external 3D fields. The MARS-Q model has been shown to well re-produce the RMP induced flow damping in MAST [26] for various $n$ numbers. The code has also recently been applied to model the flow damping due to the pure RMP fields (with $n=3$ and $n=4$ ) for one of the ITER 15MA plasmas [27]. The flow damping modelling in this study always starts with an initial rotation profile obtained from the JINTRAC modelling, with the assumption of the Prandtl number of 0.5 (cf. Fig. 1(d)).

One peculiar aspect of the initial value modelling for this 15MA plasma, is that this equilibrium, with $q_{\min }=0.95$, is unstable to the $n=1$ internal kink mode. Since MARS-Q does not have the sawtooth crash physics incorporated into the code, we shall model two possible situations. The first is the flow damping in the presence of an unstable internal kink (i.e. before 


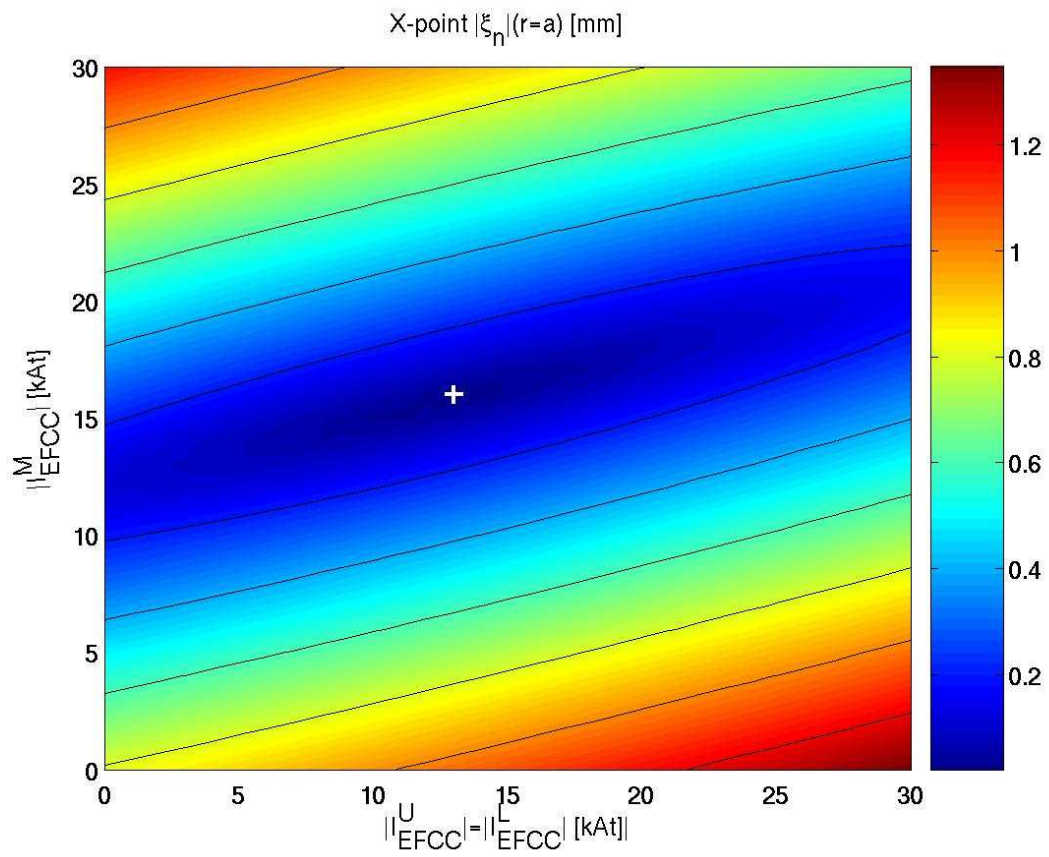

Figure 16: The computed X-point displacement of the plasma surface, with varying EFCC current amplitudes. The displacement occurs as the plasma responds to the combination of the ripple+FI+TBM fields, and to the EFCC field assuming $\Phi^{U}=\Phi^{L}=\Phi^{M}=\Phi=300^{\circ}$.

the sawtooth crash). Such a simulation will eventually lead to un-realistically large amplitude of the internal kink mode, which fully brakes the toroidal flow. The physically meaningful time period of the simulation corresponds to the stage where the amplitude of the internal kink mode still remains reasonably small.

The second situation is to model the flow damping after the sawtooth crash, where $q_{\text {min }}$ becomes slightly above 1 and the internal kink mode is stable. Indeed by slightly decreasing the total equilibrium plasma current, we can elevate the $q_{\min }$ to be slightly above 1 , thus ensuring a stable internal kink mode. Table 3 lists the linear growth rate and frequency (both normalized by the on-axis toroidal Alfvén frequency) of the MARS-F computed $n=1$ internal kink mode while scanning the $q_{\text {min }}$ value near unity. The toroidal plasma flow is included into the computation. The mode becomes marginally unstable at $q_{\min }=1.03$, and becomes stable at $q_{\min }=1.04$. The real frequency of the mode matches that of the core plasma rotation speed. In other words, the mode rotates together with the plasma.

We emphasize that changes to the original equilibrium is minimal in the scan listed in Table 3. The largest change is in the $q$-profile, which is still minor, yet the stability of the internal kink changes, which affects the flow damping modelling by MARS-Q.

We have performed the MARS-Q modelling for several choices of the $q$-profile shown in Tab. 3. Figures 17 and 18 show and compare three cases, for the perturbed resonant field amplitude and the net toroidal torques, respectively. All the $n=13 \mathrm{D}$ external fields are included. 
Table 3: Linear growth rate $\gamma$ and frequency $\omega$ of the $n=1$ internal kink mode for the $15 \mathrm{MA}$ baseline plasma.

\begin{tabular}{|r|r|r|r|}
\hline Case\# & $q_{\min }$ & $\gamma \tau_{A}$ & $\omega \tau_{A}$ \\
\hline 1 & 0.9488 & $3.67191 \mathrm{E}-3$ & $9.51908 \mathrm{E}-3$ \\
2 & 1.0057 & $4.45495 \mathrm{E}-3$ & $9.35699 \mathrm{E}-3$ \\
3 & 1.0100 & $3.99806 \mathrm{E}-3$ & $9.31278 \mathrm{E}-3$ \\
4 & 1.0200 & $2.68833 \mathrm{E}-3$ & $9.19379 \mathrm{E}-3$ \\
5 & 1.0300 & $1.03062 \mathrm{E}-3$ & $9.01798 \mathrm{E}-3$ \\
6 & 1.04 & $<0$ & - \\
\hline
\end{tabular}

Higher $q_{\min }$ generally leads to slower growth of the plasma response field, until full saturation is reached when the internal kink mode becomes marginally unstable or stable. For the three cases shown in Figs. 17 and 18, the original equilibrium, with $q_{\min }=0.95$, is most unstable to the $n=1$ internal kink, also agreeing with the computed linear growth rates of the mode shown in Tab. 3. For such cases, the non-linear runs terminate after the resonant field perturbations at rational surfaces reach too large amplitude (over 1 Gauss level) and the net toroidal torques become unrealistically large at a very short time scale (couple of milliseconds). Within this time interval, MARS-Q results show that the toroidal flow is not strongly affected. The linearly unstable internal kink mode eventually leads to numerical crash of the simulation, in the absence of additional non-linear physics associated with the sawteeth in MARS-Q.

For the fully saturated solution (where the internal kink is linearly stable), the final flow is again found to be nearly the same as the initial flow (i.e. in the absence of 3D fields). We also find that the saturated amplitude for the resonant radial field harmonics remains well below the 1 Gauss level. This is due to the strong screening of the magnetic islands by the plasma flow in the plasma core region. The saturated toroidal torques are well below $1 \mathrm{Nm}$ level even with the inclusion of the TBM field.

The periodic oscillations shown in Figs. 17 and 18 are related to the mode rotation. In fact the estimated oscillation frequency from Fig. 17 recovers well the computed mode frequency shown in Tab. 3. The oscillation frequency for the torques (Fig. 18) is roughly twice of the mode rotation frequency, as expected.

Inclusion of the $n=1$ RMP contribution from the ELM control coils almost does not affect the simulation results, for both the core flow damping and the net toroidal torques. This is because the ELM coils are configured to produce predominantly the $n=3$ field perturbation in this case. The $n=1$ side-band field is very small. The inclusion of the TBM contribution does significantly increase the torques, by a factor of about 10 , though the resulting damping is still generally too weak to substantially affect the plasma flow.

The MARS-Q modelling does not assume the equilibrium evolution. Moreover, we only model the change of the toroidal momentum due to the applied 3D fields (in our case the ripple+FI+TBM+RMP fields), by assuming that the a steady state momentum balance (essentially between the momentum source terms and the momentum diffusion term) has already 


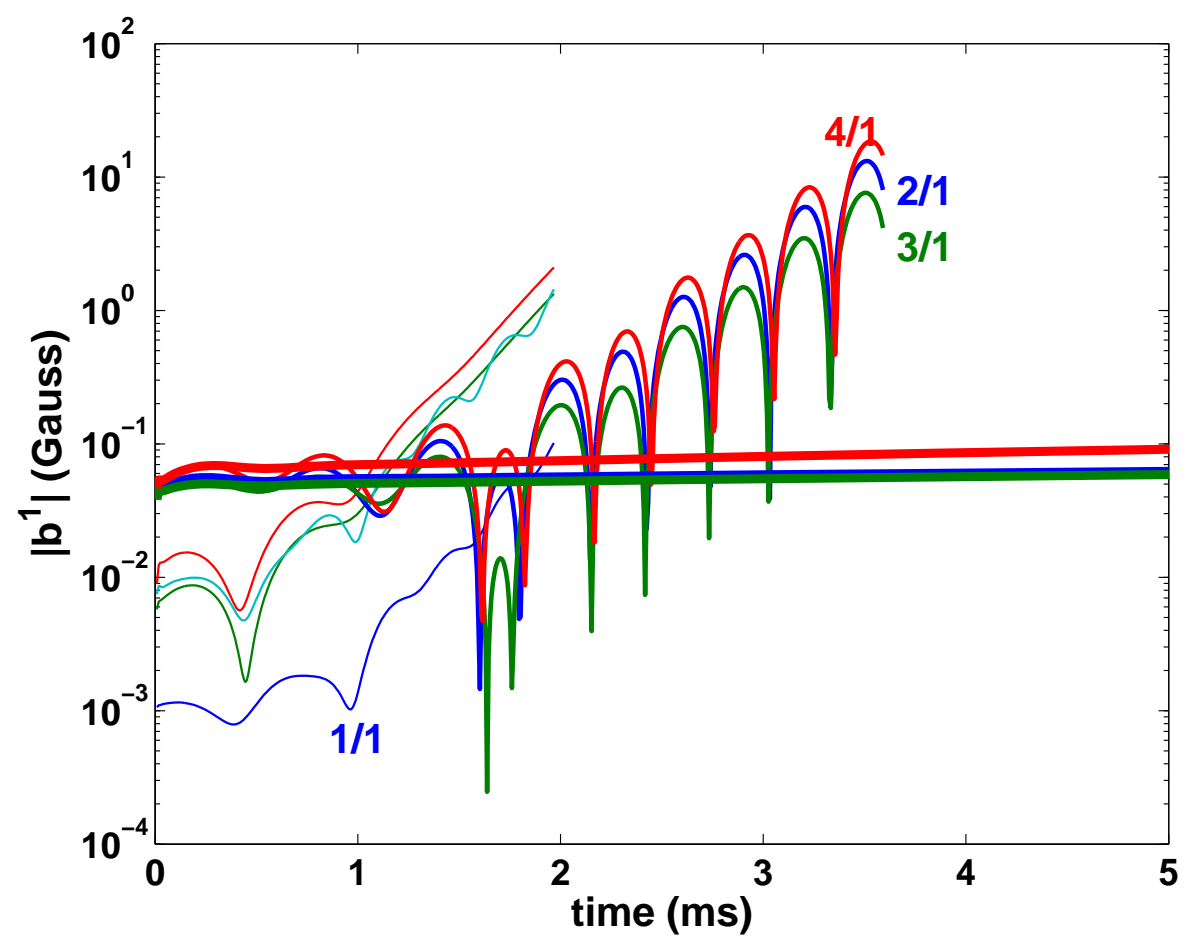

Figure 17: Time evolution of all the resonant harmonics of the radial field perturbations, computed by MARS-Q for three sets of $15 \mathrm{MA}$ baseline equilibria with $q_{\min }=0.95$ (thin lines), $q_{\mathrm{min}}=1.02$ (medium-thick lines) $q_{\mathrm{min}}=1.03$ (thick lines), as a result of quasi-linear plasma response to the $n=1$ ripple $+\mathrm{FI}+\mathrm{TBM}+\mathrm{RMP}$ fields. 


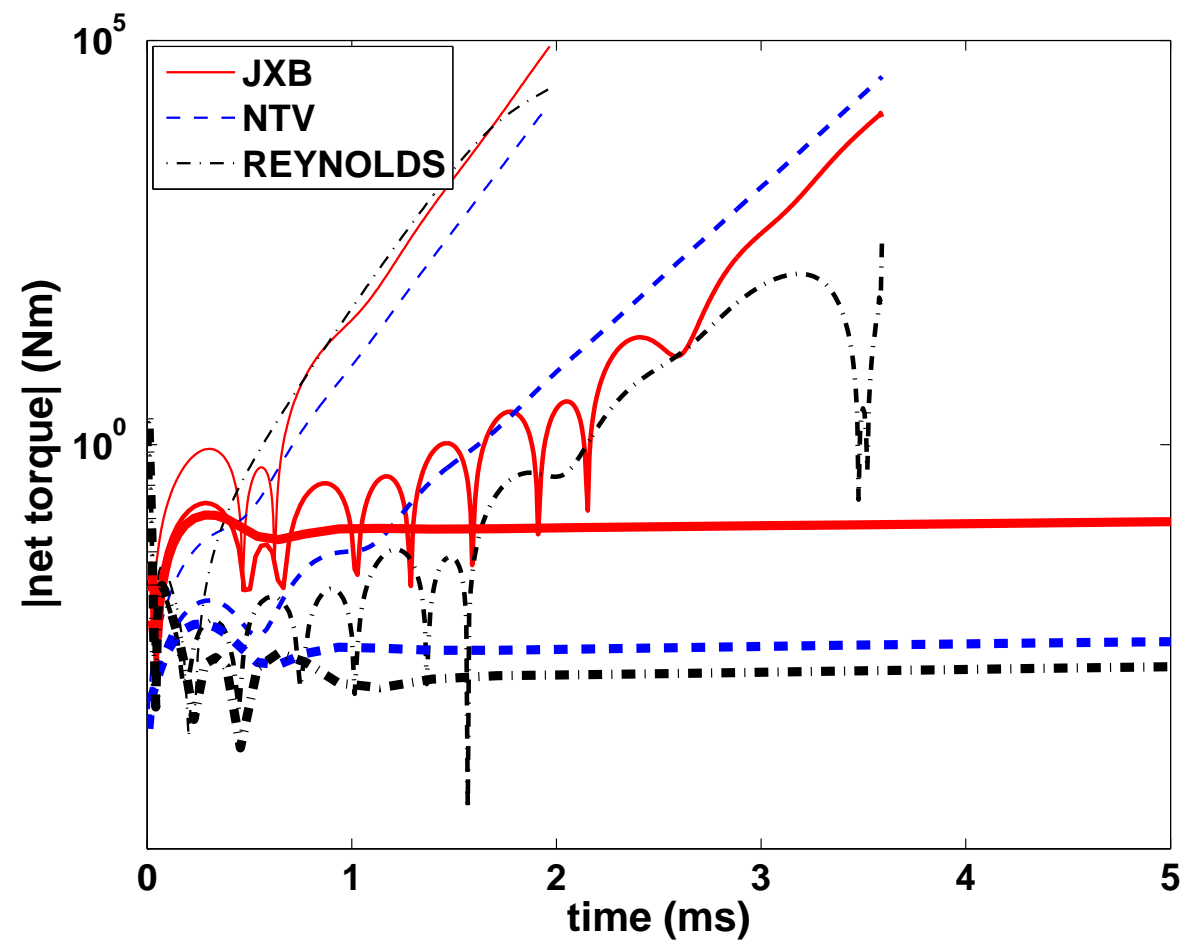

Figure 18: Time evolution of all three torque components, computed by MARS-Q for three sets of $15 \mathrm{MA}$ baseline equilibria with $q_{\mathrm{min}}=0.95$ (thin lines), $q_{\mathrm{min}}=1.02$ (medium-thick lines) $q_{\min }=1.03$ (thick lines), as a result of quasi-linear plasma response to the $n=1$ ripple+FI+TBM+RMP fields. 
been achieved before the application of the 3D fields. This allows us to avoid direct modelling of the momentum source term in the momentum evolution equation [18]. On the other hand, the JINTRAC code [19] does allow the direct modelling of both the plasma equilibrium evolution and the (time-varying) momentum source terms such as the NBI torque. But JINTRAC does not compute the toroidal torques due to the 3D fields. Therefore, we wish to couple the MARS-F and the JINTRAC codes in the following sense. We first run MARS-F to compute the linear plasma response induced toroidal torques, using the equilibrium and the flow speed as predicted by JINTRAC before the application of the 3D fields. The magnetic surface averaged torque densities due to the 3D fields are then enter into the JINTRAC transport simulation as the additional momentum sink terms. And iteration between two codes can be envisaged if necessary.

Figure 19 reports the final JINTRAC simulation results, without and with these additional torques. The predicted steady state flow profile, shown in Fig. 19(c), is almost not affected by the 3D fields induced torques, confirming the MARS-Q findings from the previous Section. There is only a slight change to the flow profile near the pedestal top. The results are probably not surprising if we compare the 3D fields induced torque with that produced by the NBI, as shown by 19(b). The NBI torque is much larger than the MARS-F computed torque due to 3D fields.

Due to the very weak effect of the 3D fields on the plasma equilibrium and the toroidal momentum evolution, there is no need to carry out an iterative procedure between MARS-F and JINTRAC, for this specific case considered in the work. Such iteration may indeed be necessary for other cases, where the $3 \mathrm{D}$ fields induced torque leads to significant modification of the plasma momentum confinement.

\section{Summary}

We have carried out computations in toroidal geometry for the ITER plasma response to 3D magnetic fields, for four scenarios: the 15MA baseline, the 12.5MA hybrid, the 9MA steady state, and finally the 7.5MA half-field Helium plasma. For the baseline and the hybrid scenarios, we also separately considered an equilibrium at the current ramp-up phase and an equilibrium during the flat-top phase.

The 3D external fields are generated by the toroidal field ripples, the ferritic inserts, the test blanket modules, the ITER error field correction coils, and in some cases also by the ELM control coils. Due to the broad toroidal spectrum of the FI and the TBM fields, we have computed the plasma response to various $n$-components of the applied fields. Since we find that the plasma response is very weak for high- $n$ field components, this allows us to limit the plasma response computations mainly for lower $n$ components, namely $n=1-6$. We have established a rigorous procedure of computing the total response field which is valid in the whole space.

A sensitivity study of the plasma response computations against the toroidal flow variation 


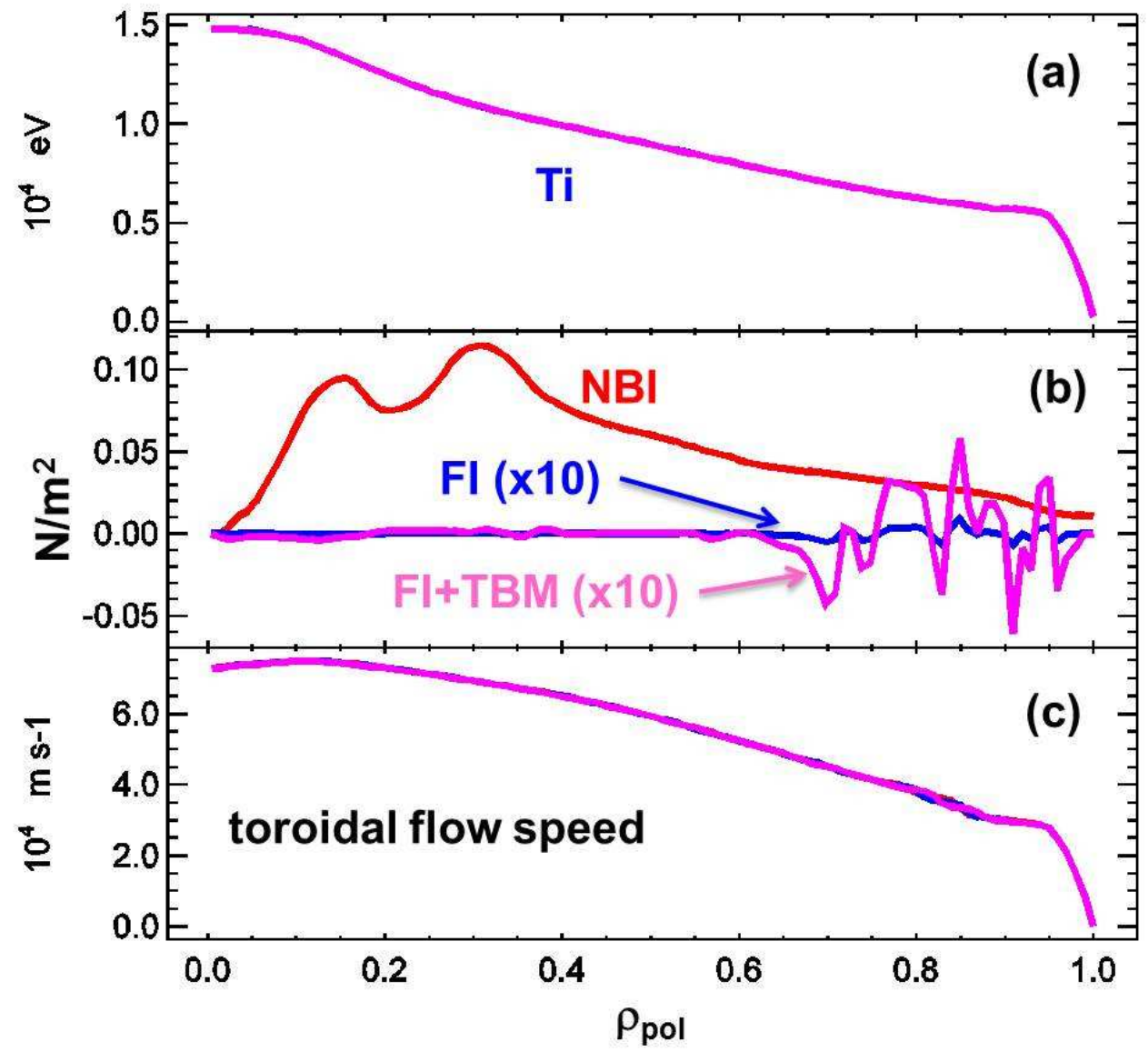

Figure 19: The JINTRAC modelled steady state radial profiles for (a) the thermal ion temperature, (b) the torque densities, and (c) the toroidal flow speed, for the 15MA baseline scenario during the current flat-top phase. Three torque densities are compared in (b): the NBI torque (red), the sum (blue) of all three torques due to plasma response to 3D fields from ripple and FI, and the sum (pink) of all three torques due to plasma response to 3D fields from ripple, FI and TBM. Three simulated steady state flow profiles are compared in (c): without the 3D fields induced torque (red), with the torque induced by ripple and FI fields (blue), with the torque induced by ripple, FI and TBM (pink). 
shows that the plasma response is not sensitive to the toroidal flow, as long as the latter does not change by order of magnitude. Based on the computed plasma response, we have also evaluated the magnetic islands width and the associated Chirikov parameter. The resistive plasma response in most cases reduces the island width compared with that of the vacuum island, and consequently, reduces the Chirikov parameter as well. Despite the fact that each individual $n$-component results in Chirikov parameter below 1, combination of all $n$ 's can result in Chirikov parameter locally exceeding 1 . The Chirikov criterion, though may still be useful as an indicator for the plasma edge field line stochastization, may be quantitatively less useful when islands with different $n$ 's overlap. A better and more direct way to judge the stochasticity is still the Poincare map. For a given $n$ ( $n=3$ and $n=4$ for ITER), the largest field perturbation, with the amplitude of the order of 100 Gauss, comes from the ELM control coils (the RMP fields). On the other hand, the symmetry of the ELM coils in ITER normally generates narrow band toroidal spectrum of the 3D field perturbation, compared to the rather broad spectrum (for $n$ up to 20) generated by the ripple, FI, and particularly the ITER TBM, though the corresponding $n=3$ and $n=4$ components of these 3D broadband fields are about ten times smaller than the ELM fields. The amplitude of the TBM field is typically several times larger than that of the FI field. Therefore, most of the broadband 3D fields in ITER are produced by the TBM components. On the other hand, the toroidal and poloidal spectra of the fields are different between these two components.

Based on the plasma response computations, we also performed a study on optimal error field correction using the ITER EFCC, where the error field is the assumed to be the $n=1$ component of the ripple field, the FI and the TBM field. The study is made based on the 15MA baseline case during the current flat-top phase. The EFC optimizations have been carried out using various optimization criteria designed for an early study on the EFC in MAST [21]. The optimizing parameters are both the amplitude and the toroidal phase of the EFCC currents flowing in the top, middle, and the bottom rows. The key finding is that the middle row of EFCC plays the dominant role in correcting the EF due to ripple+FI+TBM. At a fixed coil current amplitude, it turns out that about $300^{\circ}$ is the optimal phase for the middle row of EFCC current, in order to correct all the 3D fields including that of the (dominant) TBM contribution. This is robustly predicted by all but the vacuum field based optimization criteria considered in this work, as well as by various choices of the coil current amplitude.

Both the MARS-Q modelling and the JINTRAC modelling, to which the 3D fields induced torques are provided by the MARS-F computation, show negligible flow damping by the $n=$ 1 component of all the 3D fields considered in this work, for the 15MA baseline plasma. In addition, MARS-Q modelling also shows that, in the absence of the $n=1$ internal kink instability, the non-linear time evolution fully saturates after about $100 \mathrm{~ms}$. The dominant torque is provided by the resonant electromagnetic torque. The JINTRAC modelling shows that the total torque due to all the $n=13 \mathrm{D}$ fields, even in the presence of TBM field, is still at least one order of magnitude smaller than the NBI torque, for the 15MA baseline scenario.

Although not included in this work, we mention that similar MARS-Q and JINTRAC runs have also been performed for the 9MA steady state plasma, with very similar findings for the flow damping. The $n=1$ fields from FIs and TBMs do not provide appreciable change to the toroidal flow. 


\section{Appendix: Magnetic island width and Chirikov parameter}

\section{Evaluation of island width in MARS-F coordinates}

In MARS-F PEST-like coordinates $(s, \chi, \phi)$, defining $\chi_{m n} \equiv m \chi+n q$ and $\delta s$ as the variation of $s$ of the 3D field line along the helical angle $\chi_{m n}$, we have

$$
\frac{\partial \delta s}{\partial \chi_{m n}}=\frac{\mathbf{B} \cdot \nabla s}{\mathbf{B} \cdot \nabla \chi_{m n}}
$$

where $\mathbf{B}=\mathbf{B}_{e q}+\mathbf{b}$ is the total 3D magnetic field, and we shall only consider one resonant harmonic for the perturbed field $\mathbf{b}$. In MARS-F formulation, we have

$$
\begin{aligned}
\mathbf{B} \cdot \nabla s & =\mathbf{b} \cdot \nabla s=J^{-1} Q e^{i \chi_{m n}}, \quad Q \equiv b_{\mathrm{MARS}-\mathrm{F}}^{1 m n} \\
\mathbf{B} \cdot \nabla \chi_{m n} & \simeq \mathbf{B}_{e q} \cdot \nabla \chi_{m n}=\mathbf{B}_{e q} \cdot(m \nabla \chi+n \nabla \phi) \\
& =m \hat{\psi}^{\prime} J^{-1}+n F / R^{2}=\hat{\psi}^{\prime} J^{-1}(m+n q) \simeq \hat{\psi}^{\prime} J^{-1} n q^{\prime} \delta s,
\end{aligned}
$$

where $\hat{\psi}^{\prime} \equiv d \hat{\psi} / d s$, and we have used the fact that in the PEST-like straight field line coordinate system,

$$
q=\frac{F / R^{2}}{\hat{\psi}^{\prime} / J} \simeq q_{s}+q^{\prime} \delta s
$$

Thus we have

$$
\frac{\partial \delta s}{\partial \chi_{m n}}=\frac{Q}{n \hat{\psi}^{\prime} q^{\prime} \delta s} e^{i \chi_{m n}}
$$

or

$$
\frac{\partial(\delta s)^{2}}{\partial \chi_{m n}}=\frac{2 Q}{n \hat{\psi}^{\prime} q^{\prime}} e^{i \chi_{m n}}
$$

yielding

$$
(\delta s)^{2}=\left.\frac{2 Q}{i n \hat{\psi}^{\prime} q^{\prime}} e^{i \chi_{m n}}\right|_{\chi_{1}} ^{\chi_{2}}, \quad(\delta s)_{\max }^{2}=\frac{4|Q|}{\left|n \hat{\psi}^{\prime} q^{\prime}\right|}, \quad|\delta s|_{\max }=2 \sqrt{\left|\frac{Q}{n \hat{\psi}^{\prime} q^{\prime}}\right|} .
$$

Finally we define the island width, in terms of the normalised minor radius $s$, to be

$$
w=2|\delta s|_{\max }=4 \sqrt{\left|\frac{Q}{n \hat{\psi}^{\prime} q^{\prime}}\right|}=4 \sqrt{\left|\frac{s Q}{n \hat{\psi}^{\prime} q S}\right|}=4 \sqrt{\left|\frac{1}{2 \hat{\psi}_{0}} \frac{Q}{n q S}\right|},
$$

where $S \equiv s d q / d s / q$ as calculated at the rational surface, and we have used the fact that $\hat{\psi}^{\prime}=2 \hat{\psi}_{0} s$. 


\section{An equivalent approach for evaluation of island width}

Following Ref. [36], the island width, in toroidal geometry and in terms of the normalized poloidal flux $\psi$, is calculated as

$$
\begin{aligned}
& w_{\psi}=\delta \psi=4 \sqrt{\frac{b_{m n}}{n q^{\prime}}}, \quad q^{\prime}=\frac{d q}{d \psi}, \quad \psi=\frac{\Psi_{p}-\psi_{\text {axis }}}{\Psi_{0}}, \\
& b_{m n}=\frac{\delta B_{m n} A}{2 \pi \Psi_{0}}, \quad \delta B_{m n} A=2 \oint \cos (m \theta-n \phi) \delta \mathbf{B} \cdot d \mathbf{S},
\end{aligned}
$$

where $\Psi_{0} \equiv \psi_{\text {sep }}-\psi_{\text {axis }}=2 \pi \hat{\psi}_{0}$. Note the factor of $2 \pi$ here due to special definition of the poloidal flux in MARS-F.

In terms of MARS-F variables, $d \mathbf{S} \equiv J_{s} \hat{\mathbf{n}} d \chi d \phi=J \nabla s d \chi d \phi$, we obtain

$$
\delta B_{m n} A=\oint e^{-(i m \chi+i n \phi)} J \delta \mathbf{B} \cdot \nabla s d \chi d \phi=(2 \pi)^{2} Q
$$

where as before, $Q \equiv b_{\text {MARS-F. }}^{1 m n}$. Note that the factor of 2 in the definition of $\delta B_{m n} A$ disappears due to different Fourier representations for the perturbed field. We thus obtain

$$
b_{m n}=\frac{Q}{\hat{\psi}_{0}}
$$

Since $\psi=s^{2}$, we have

$$
q^{\prime}=\frac{d q}{d \psi}=\frac{d q}{d s} \frac{1}{2 s}
$$

Substituting all the above factors into (19), we obtain the island width in terms of $\psi$

$$
w_{\psi}=\delta \psi=4 \sqrt{\frac{2 s^{2}}{\hat{\psi}_{0}} \frac{Q}{n q S}}, \quad S \equiv \frac{s d q / d s}{q} .
$$

Since $\psi=s^{2}$, we have $\delta \psi=2 s \delta s$. Therefore, the island width, in terms of $s$, is

$$
w=4 \sqrt{\left|\frac{1}{2 \hat{\psi}_{0}} \frac{Q}{n q S}\right|},
$$

which is exactly the same as that calculated in Eq. (18).

In case the island is generated by the combination of both $(m, n)$ and $(-m,-n)$ resonant harmonics, the factor $Q$ should be re-defined as

$$
Q=\operatorname{Re}\left[b_{\text {MARS-F }}^{1 m n}+b_{\text {MARS-F }}^{1,-m,-n}\right]=2 \operatorname{Re}\left[b_{\text {MARS-F }}^{1 m n}\right] .
$$




\section{Chirikov parameter}

The Chirikov parameter, between two adjacent islands located at rational surfaces $s_{1}$ and $s_{2}$, is defined as

$$
\sigma=\frac{w_{1}+w_{2}}{2\left|s_{2}-s_{1}\right|}
$$

This parameter can also be approximately defined for a single island, assuming a single $n$ field perturbation. In this case, the distance $\Delta s$ between two adjacent rational surfaces is approximately calculated via

$$
q\left(s_{m+1}\right)-q\left(s_{m}\right)=q^{\prime} \Delta s
$$

Since $n q\left(s_{m+1}\right)-n q\left(s_{m}\right)=(m+1)-m=1$, we have $\Delta s=1 /\left(n q^{\prime}\right)$. Thus

$$
\sigma=\frac{w}{\Delta s}=4 \sqrt{\left|\frac{1}{2 \hat{\psi}_{0}} \frac{n q S Q}{s^{2}}\right|} .
$$

\section{An asymptotic scaling of Chirikov parameter at large $n$}

Consider a large-aspect-ratio model for the perturbed vacuum field $\mathbf{b}=\nabla \tilde{\psi}$, satisfying

$$
\nabla \cdot \mathbf{b}=\nabla^{2} \tilde{\psi}=\frac{1}{r} \frac{\partial}{\partial r}\left(r \frac{\partial \tilde{\psi}}{\partial r}\right)+\frac{1}{r^{2}} \frac{\partial^{2} \tilde{\psi}}{\partial \theta^{2}}+\frac{\partial^{2} \tilde{\psi}}{\partial z^{2}}=0 .
$$

Assuming $\tilde{\Psi}=\psi(r) e^{i m \theta+i k z}$, with $k=n / R_{0}$, we obtain the modified Bessel's equation

$$
x^{2} \frac{\partial^{2} \psi}{\partial x^{2}}+x \frac{\partial \psi}{\partial x}-\left(x^{2}+m^{2}\right) \psi=0
$$

where $x \equiv n r / R_{0}$. The physically interesting solution is the modified Bessel function $I_{m}(x)$

$$
I_{m}(x)=\sum_{j=0}^{\infty} \frac{1}{j ! \Gamma(j+m+1)}\left(\frac{x}{2}\right)^{2 j+m} .
$$

At small argument $0<x<<\sqrt{m+1}$, we have

$$
I_{m}(x) \simeq \frac{1}{\Gamma(m+1)}\left(\frac{x}{2}\right)^{m} .
$$

At large argument, we have

$$
I_{m}(x) \simeq \frac{e^{x}}{\sqrt{2 \pi x}}
$$


We shall consider the large argument asymptote, assuming $n$ is large. Assuming that the vacuum magnetic field amplitude scales as $1 / n$ at the plasma boundary, i.e. $Q(r=a)=1 / n$, where

$$
Q(r)=C_{0} \frac{e^{n r / R_{0}}}{\sqrt{n r / R_{0}}} .
$$

The boundary condition gives $C_{0}=\sqrt{a / R_{0}} e^{-n a / R_{0}} / \sqrt{n}$, thus

$$
Q(r)=\frac{1}{n} \frac{1}{\sqrt{s}} e^{-n \varepsilon_{0}(1-s)},
$$

where $\varepsilon_{0} \equiv a / R_{0}, s \equiv r / a$. Inserting the above equation into (29), we obtain the large- $n$ scaling for the Chirikov parameter

$$
\sigma \sim s^{-5 / 4} e^{-n \varepsilon_{0}(1-s) / 2}
$$

showing that the Chirikov parameter decays exponentially at large $n$, basically due to the fact that the vacuum field amplitude decays exponentially with $n$.

\section{Acknowledgements}

This work was funded by the F4E Contract F4E-OPE-650. The work was also part funded by the RCUK Energy Programme under grant EP/I501045, as well as by National Natural Science Foundation of China (NSFC) [grant numbers 11428512 and 11405029] and National Magnetic Confinement Fusion Science Program under grant No. 2015GB104004. The supercomputing resources of CSC-IT center for science, Finland, were utilized in the studies. The work was also partially funded by the Academy of Finland project No. 259675. The views and opinions expressed herein do not necessarily reflect those of the European Commission.

\section{References}

[1] J.-S. Lönnroth et al., Plasma Phys. Control. Fusion 49, 273 (2007).

[2] P.C. de Vries el al., Nucl. Fusion 48, 035007 (2008).

[3] J.A. Snipes et al., "Results of ITER Test Blanket Module Mock-up Experiments on DIIID”, Proc. 37th EPS Conf. on Plasma Physics (Dublin, 2010) Vol. 34A (ECA) P1.1093 (2010).

[4] M.J. Schaffer el al., Nucl. Fusion 51, 103028 (2011).

[5] J. Varje et al., "Effect of plasma response on the fast ion losses due to ELM control coils in ITER", Nucl. Fusion, in press (2016).

[6] J.-K. Park el al., Nucl. Fusion 51, 023003 (2011). 
[7] M.J. Lanctot et al., Phys. Plasmas 17, 030701 (2010).

[8] M.J. Lanctot et al., Phys. Plasmas 18, 056121 (2011).

[9] A.D. Turnbull et al., Phys. Plasmas 20, 056114 (2013).

[10] S. Haskey et al., Plasma Phys. Control. Fusion 57, 025015 (2015).

[11] J.D. King et al., Phys. Plasmas 22, 072501 (2015).

[12] Y.Q. Liu el al., Nucl. Fusion 51, 083002 (2011).

[13] D. Ryan et al., Plasma Phys. Control. Fusion 57, 095008 (2015).

[14] Z.R. Wang et al., Phys. Rev. Lett. 114, 145005 (2015).

[15] N.M. Ferraro et al., Phys. Plasmas 19, 056105 (2012).

[16] Y.Q. Liu et al., Phys. Plasmas 7, 3681 (2000).

[17] Y.Q. Liu et al., Phys. Plasmas 15, 112503 (2008).

[18] Y.Q. Liu et al., Phys. Plasmas 20, 042503 (2013).

[19] M. Romanelli et al., Plasma and Fusion Research 9, 3403023 (2014).

[20] A. Bondeson and R. Iacono, Phys. Fluids B 1, 1431 (1989).

[21] Y.Q. Liu et al., Plasma Phys. Control. Fusion 56, 104002 (2014).

[22] Y.Q. Liu et al., Phys. Plasmas 21, 056105 (2014).

[23] J.W. Berkery et al., Phys. Plasmas 21, 052505 (2014).

[24] J.E. Menard et al., Phys. Rev. Lett. 113, 255002 (2014).

[25] Z.R. Wang et al., Phys. Plasmas 21, 042502 (2014).

[26] Y.Q. Liu et al., Plasma Phys. Control. Fusion 54, 124013 (2012).

[27] Y.Q. Liu el al., Nucl. Fusion 55, 063027 (2015).

[28] S. Haskey et al., Plasma Phys. Control. Fusion 56, 035005 (2014).

[29] S. Äkäslompolo et al., Fusion Eng. Design 98-99, 1039 (2015).

[30] Y.Q. Liu et al., Phys. Plasmas 15, 072516 (2008).

[31] Y.Q. Liu et al., Plasma Phys. Control. Fusion 51, 115006 (2009).

[32] T.E. Evans et al., Nucl. Fusion 53, 093029 (2013).

[33] Y.Q. Liu et al., Phys. Plasmas 17, 122502 (2010).

[34] R.J. Buttery et al., Nucl. Fusion 51, 073016 (2011).

[35] ITER Document: ITER_D_2EP2GB v1.2 (2008).

[36] I. Joseph, et al., Nucl. Fusion 48, 045009(2008). 